\title{
*-DIFFERENTIAL IDENTITIES OF PRIME RINGS WITH INVOLUTION
}

\author{
CHEN-LIAN CHUANG
}

\begin{abstract}
Main Theorem. Let $R$ be a prime ring with involution * . Suppose that $\phi\left(x_{i}^{\Delta_{j}},\left(x_{i}^{\Delta_{j}}\right)^{*}\right)=0$ is a $*$-differential identity for $R$, where $\Delta_{j}$ are distinct regular words of derivations in a basis $M$ with respect to a linear order $<$ on $M$. Then $\phi\left(z_{i j}, z_{i j}^{*}\right)=0$ is a *-generalized identity for $R$, where $z_{i j}$ are distinct indeterminates.
\end{abstract}

Along with the Main Theorem above, we also prove the following:

Proposition 1. Suppose that ${ }^{*}$ is of the second kind and that $C$ is infinite. Then $R$ is special.

Proposition 2. Suppose that $S_{W}(V) \subseteq R \subseteq L_{W}(V)$. Then $Q$, the two-sided quotient ring of $R$, is equal to $L_{W}(V)$.

Proposition 3 (Density theorem). Suppose that ${ }_{D} V$ and $W_{D}$ are dual spaces with respect to the nondegenerate bilinear form $($,$) . Let v_{1}, \ldots, v_{s}, v_{1}^{\prime}, \ldots$, $v_{s}^{\prime} \in V$ and $u_{1}, \ldots, u_{t}, u_{1}^{\prime}, \ldots, u_{t}^{\prime} \in W$ be such that $\left\{v_{1}, \ldots, v_{s}\right\}$ is $D$ independent in $V$ and $\left\{u_{1}, \ldots, u_{t}\right\}$ is D-independent in $W$. Then there exists $a \in S_{W}(V)$ such that $v_{i} a=v_{i}^{\prime} \quad(i=1, \ldots, s)$ and $a^{*} u_{j}=u_{j}^{\prime} \quad(j=1, \ldots, t)$ if and only if $\left(v_{i}^{\prime}, u_{j}\right)=\left(v_{i}, u_{j}^{\prime}\right)$ for $i=1, \ldots, s$ and $j=1, \ldots, t$.

Proposition 4. Suppose that $R$ is a prime ring with involution * and that $f$ is a *-generalized polynomial. If $f$ vanishes on a nonzero ideal of $R$, than $f$ vanishes on $Q$, the two-sided quotient ring of $R$.

The objective of this paper is to prove the following generalization of Kharchenko's theorem on differential identities to prime rings with involution (the notation here is explained in $\S I$ below):

Main Theorem. Let $R$ be a prime ring with involution ${ }^{*}$. Suppose that $\phi\left(x_{i}^{\Delta_{j}},\left(x_{i}^{\Delta_{j}}\right)^{*}\right)=0$ is a $*$-differential identity for $R$, where $\Delta_{j}$ are distinct regular words of derivations in a basis $M$ with respect to a linear order $<$ on $M$. Then $\phi\left(z_{i j}, z_{i j}^{*}\right)=0$ is a *-generalized identity for $R$, where $z_{i j}$ are distinct indeterminates.

Received by the editors April 18, 1988.

1980 Mathematics Subject Classification (1985 Revision). Primary 16A38; Secondary 16A28, 16A72, 16A12, 16A48.

Key words and phrases. Differential identity, generalized (polynomial) identity, left (Martindale) quotient ring, two-sided (Martindale) quotient ring, prime rings with involution. 
We have the following immediate

Corollary. Let $R$ be a prime ring with involution * . Then any *-differential identity of $R$ is a consequence of the basic identities (1)-(9) of §I and *generalized identities of $R$.

There is a similar work [5] by Lanski. However the main result (Theorem 7) of [5] is false. Before proceeding, it seems proper to give the following counterexample to Theorem 7 [5] (and Theorem 4 [5] as well):

Counterexample. (Using Lanski's type of notation [5].) Let $Q$ be the field of rational numbers and let $F=Q(t)$, the field of rational functions in $t$ with coefficients in $Q$. Set $R=F_{n}$, the ring of all $n \times n$ matrices over $F$, and endow $R$ with the transpose involution ${ }^{*}$; that is, for $x=\left(\alpha_{i j}\right) \in R, x^{*}=\left(\alpha_{j i}\right)$. Define the derivation $\delta$ on $R$ by setting, for $x=\left(\alpha_{i j}\right) \in R, x^{\delta}=\left((d / d t) \alpha_{i j}\right)$, where $d / d t$ is the usual differentiation derivative with respect to $t$. Observe that $\left(x^{*}\right)^{\delta}=\left(x^{\delta}\right)^{*}$ for $x \in R$.

Let $e_{i j}(i, j=1, \ldots, n)$ be the matrix units of $R$; that is, let $e_{i j}$ be the matrix of $R$ with 1 in the $(i, j)$ entry and zero elsewhere. Let

$$
b=\sum_{i=1}^{n} i e_{i i}=\left(\begin{array}{cccc}
1 & & & 0 \\
& 2 & & \\
& & \ddots & \\
0 & & & n
\end{array}\right) \text {. }
$$

Define the derivation $d$ on $R$ by setting $x^{d}=x^{\delta}+[b, x]$ for $x \in R$. Now consider $s=x^{d}+\left(x^{*}\right)^{d}-\left[2 b, x^{*}\right]$ for $x \in R$. We compute $s=x^{d}+\left(x^{*}\right)^{d}-$ $\left[2 b, x^{*}\right]=x^{\delta}+[b, x]+\left(x^{*}\right)^{\delta}+\left[b, x^{*}\right]-2\left[b, x^{*}\right]=x^{\delta}+[b, x]+\left(x^{\delta}\right)^{*}-\left[b, x^{*}\right]=$ $x^{\delta}+\left(x^{\delta}\right)^{*}+\left[b, x-x^{*}\right]$. So $s$ is always symmetric and hence $e_{11} s e_{21}-e_{12} s e_{11}=$ 0 . We have thus shown that

$$
f(x, y)=e_{11}\left(x^{d}+y^{d}-[2 b, y]\right) e_{21}-e_{12}\left(x^{d}+y^{d}-[2 b, y]\right) e_{11}
$$

is a $G^{*}$-DI of $R$ in the sense of [5]. The derivation $d$ is obviously outer and can be chosen to be the first element in the well-ordering of the basis of the space of derivations on $R$ modulo the space of inner derivations (that is, $M \backslash M_{0}$ in [5]). Let $\varnothing$ denote the empty set. Theorem 7 [5] asserts that $f_{\varnothing}(x, y)$ $=e_{12}[2 b, y] e_{11}-e_{11}[2 b, y] e_{21}$ is a $G^{*}$-PI of $R$. But, since $f_{\varnothing}\left(e_{12}, e_{12}^{*}\right)=$ $f_{\varnothing}\left(e_{12}, e_{21}\right)=2 e_{21} \neq 0$, this is obviously false!

The crucial difference between Lanski's Theorem 7 [5] and our Main Theorem above is as follows: In [5], ${ }^{*}$ remains inside of derivation words. By introducing $d^{*}$ for each derivation $d$ on $R$, we are able to push ${ }^{*}$ outside of the derivation words in our Main Theorem and this gives the right form for doing induction.

Ignoring the falsity of [5], our improvement is to remove the multilinearity assumption of [5]. Viewing the complexity of Kharchenko's analogous work [4] for rings without involution, this part is quite intricate. For this work, a 
structure theory for $*$-primitive rings with socle is required and this is developed in $\S I I$. There, as auxiliary results, we obtain an interesting characterization of the Martindale two-sided quotient ring and we also prove the useful *-version of the Jacobson density theorem.

One minor difference worth emphasizing is that the coefficients of our *differential identities must be allowed to lie in the left Martindale quotient ring of $R$, instead of the two-sided Martindale quotient ring as in Lanski [5]. This is not simply a matter of generality and is actually crucial to our proof, as will be seen in §IV.

Our method is by mixing Kharchenko's techniques with a theorem due to Rowen. The material is organized as follows: In $\S I$, we generalize Kharchenko's original theorem slightly and meanwhile explain our notations. In §II, we treat the case when ${ }^{*}$ is of the second kind and introduce the notion of special differential identities. In $\S I I I$, we generalize those well-known results on rings with nonzero socle to their *-versions. The proof of the Main Theorem is given in $\S I V$.

Along with proving the Main Theorem above, the interesting auxiliary results obtained are singled out under the title "Proposition".

Most of the notation here is adopted from Kharchenko's original work [3, 4] instead of Lanski [5].

\section{Preliminaries}

Suppose that $R$ is a prime ring and that $F$ is the set of all its nonzero ideals. Let $R_{F}$ be the ring of left quotients of $R$ relative to $F$; that is, $R_{F}=$ $\stackrel{\lim }{\rightarrow} \operatorname{Hom}\left({ }_{R} I, R\right)$. Consider the subring $Q$ of $R_{F}$ consisting of those elements $\overrightarrow{a \in R_{F}}$ for which there exists a nonzero ideal $I_{a} \in F$ such that $a I_{a} \subseteq R . Q$ is called the two-sided quotient ring of $R$ relative to $F$. The center of $Q$, denoted by $C$, coincides with the center of $R_{F}$ and is called the extended centroid of $R$.

Let $\operatorname{Der} Q$ consist of all derivations on $Q$. For $\sigma \in \operatorname{Der} Q$ and $\alpha \in C$, define $\sigma \alpha$ by $x^{\sigma \alpha}=\left(x^{\sigma}\right) \alpha$ for $x \in Q$. For $\sigma, \mu \in \operatorname{Der} Q$, define $x^{[\sigma, \mu]}=$ $\left(x^{\sigma}\right)^{\mu}-\left(x^{\mu}\right)^{\sigma}$ for $x \in Q . \sigma \alpha$ and $[\sigma, \mu]$ thus defined are also derivations of $Q$. In this manner, $\operatorname{Der} Q$ is a right vector space over $C$ and is also a Lie algebra (over the subfield consisting of $\alpha \in C$ such that $\alpha^{\sigma}=0$ for all $\sigma \in \operatorname{Der} Q)$. Der $Q$ is called the differential Lie C-algebra of derivations in $Q$. Let $\operatorname{Der} R$ consist of all derivations on $R$ and let $D=(\operatorname{Der} R) \cdot C$. Obviously $D \subseteq \operatorname{Der} Q$.

By a differential polynomial, we mean a generalized polynomial involving noncommutative indeterminates which are acted by derivations of $R$ as unary operations. We allow the coefficients of a differential polynomial to lie in $R_{F}$. Obviously, every differential polynomial can be written in the form $\phi\left(x_{i}^{\Delta_{j}}\right)$, where $\phi\left(z_{i j}\right)$ is an ordinary generalized polynomial in $z_{i j}$ and $\Delta_{j}$ are words of derivations of $R, \phi=0$ is said to be a differential identity for $R$ if 
$\phi$ assumes the constant value 0 for any assignment of values from $R$ to its indeterminates.

The following basic differential identities hold in any prime rings:

(1) $(x y)^{\sigma}=x^{\sigma} y+x y^{\sigma}$, where $\sigma \in \operatorname{Der} Q$.

(2) $(x+y)^{\sigma}=x^{\sigma}+y^{\sigma}$, where $\sigma \in \operatorname{Der} Q$.

(3) $x^{\sigma}=x a-a x$, where $\sigma$ is the inner derivation defined by $a \in Q$.

(4) $x^{[\sigma, \mu]}=\left(x^{\sigma}\right)^{\mu}-\left(x^{\mu}\right)^{\sigma}$, where $\sigma, \mu \in \operatorname{Der} Q$ and $[\sigma, \mu]$ is their commutator.

(5) $\left(\cdots\left(\left(x^{\sigma}\right)^{\sigma}\right) \cdots\right)^{\sigma}=x^{\left(\sigma^{p}\right)}$, where $\sigma \in \operatorname{Der} Q$ and where $p$ is the characteristic of the given ring. If $p=0$, then this identity assumes the form $x=x$.

(6) $x^{\sigma \alpha+\mu \beta}=\left(x^{\sigma}\right) \alpha+\left(x^{\mu}\right) \beta$, where $\sigma, \mu \in \operatorname{Der} Q$ and $\alpha, \beta \in C$.

Let $D_{\text {int }}$ be the Lie subalgebra of $\operatorname{Der} Q$ consisting of all inner derivations of $Q$. We choose a basis $M_{0}$ for the right $C$-vector space $D_{\text {int }}$ and augment it to a basis $M$ of $D_{\text {int }}+D$. We also fix a total order $>$ in the set $M$ such that $\mu_{0}>\mu$ for $\mu_{0} \in M_{0}$ and $\mu \in M \backslash M_{0}$. We then extend this order to the set of all derivation words in $M$ by assuming that a longer word is greater than a shorter one and that words of the same length are ordered lexicographically. By a regular word in $M$, we mean a word of the form $\Delta=\delta_{1}^{s_{1}} \delta_{2}^{s_{2}} \cdots \delta_{m}^{s_{m}}$ such that

(1) $\delta_{i} \in M \backslash M_{0}$ for $i=1, \ldots, m$,

(2) $\delta_{1}<\delta_{2}<\cdots<\delta_{m}$, and

(3) $s_{i}<p$ for $i=1, \ldots, m$, if the characteristic of $R$ is $p>0$.

By means of the basic identities (1)-(6), any differential identity can be transformed into the form $\phi\left(x_{i}^{\Delta_{j}}\right)=0$ where

(1) $\phi\left(z_{i j}\right)$ is a generalized polynomial in distinct indeterminates $z_{i j}$, and where

(2) $\Delta_{j}$ are regular words in $M$.

Kharchenko [4] has proved the following (actually for semiprime rings with characteristic):

Theorem (Kharchenko). Let $R$ be a prime ring. Suppose that $\phi\left(x_{i}^{\Delta_{\prime}}\right)=0$ is a differential identity for $R$, where $\Delta_{j}$ are distinct regular words. Then $\phi\left(z_{i j}\right)=0$ is a generalized identity for $R$, where $z_{i j}$ are distinct indeterminates.

Now suppose that $R$ is a prime ring endowed with the involution ${ }^{*}$. Note that ${ }^{*}$ can be uniquely extended to $Q$ in the following manner: Let $a \in Q$ and let $I \in F$ be such that $I a \subseteq R$ and $a I \subseteq R$. Define $a^{*}$ by the following: $a^{*}:{ }_{R} I^{*} \rightarrow{ }_{R} R$ via $r^{*} a^{*}=(a r)^{*}$. It is obvious that $a^{*} \in R_{F}$. We can verify easily that for $r \in I, a^{*} r^{*}=(r a)^{*} \in R$. So $a^{*} \in Q$ as desired. We assume henceforth that ${ }^{*}$ is defined on the whole $Q$. We say that ${ }^{*}$ is of the first kind if $\alpha^{*}=\alpha$ for all $\alpha \in C$. Otherwise, we say that ${ }^{*}$ is of the second kind. 
By a $*$-differential polynomial, we mean a generalized polynomial involving noncommutative indeterminates which are acted by the involution ${ }^{*}$ as well as derivations of $R$, regarded as unary operations. Although, in general, ${ }^{*}$ cannot be extended to $R_{F}$, we still allow the coefficients of a *-differential polynomial to lie in $R_{F}$. The following is an example of a $*$-differential polynomial:

$$
\phi(x, y)=a_{1} x^{* \delta_{1} *} a_{2} y^{\delta_{2} *} a_{3}+b_{1} y b_{2} y^{\delta_{2}} x^{*} b_{3},
$$

where $a_{1}, a_{2}, a_{3}, b_{1}, b_{2}, b_{3} \in R_{F}$ and $\delta_{1}, \delta_{2} \in D_{\text {int }}+D$.

For a $*$-differential polynomial $\phi$, we say that $\phi=0$ is a $*$-differential identity for $R$ if $\phi$ assumes the constant value 0 for any assignment of values from $R$ to its indeterminates.

The following basic identities are trivial for any rings with involution ${ }^{*}$ :

(7) $(x y)^{*}=y^{*} x^{*}$.

(8) $(x+y)^{*}=x^{*}+y^{*}$.

For $\sigma \in \operatorname{Der} Q$, we define $\sigma^{*}$ as follows: For $x \in Q, x^{\left(\sigma^{*}\right)}=\left(\left(x^{*}\right)^{\sigma}\right)^{*}$.

It is easy to verify that $\sigma^{*}$, thus defined, is also a derivation of $Q$. The following basic $*$-differential identity holds in any rings with involution ${ }^{*}$ :

(9) $\left(x^{\sigma}\right)^{*}=\left(x^{*}\right)^{\left(\sigma^{*}\right)}$, where $\sigma \in \operatorname{Der} Q$.

An immediate generalization of $(9)$ is the following:

$(9)^{\prime}\left(x^{\delta_{1} \cdots \delta_{n}}\right)^{*}=\left(x^{*}\right)^{\left(\delta_{1}^{*}\right)\left(\delta_{2}^{*}\right) \cdots\left(\delta_{n}^{*}\right)}$, where $\delta_{1}, \delta_{2}, \ldots, \delta_{n} \in \operatorname{Der} Q$.

By means of $(9)^{\prime}$, we can push ${ }^{*}$ inward or outward through derivations. Bringing ${ }^{*}$ outside of derivations, every $*$-differential identity can be transformed to the form $\phi\left(x_{i}^{\Delta_{j}},\left(x_{i}^{\Delta_{j}}\right)^{*}\right)=0$, where $\Delta_{j}$ are words of derivations only, not containing any ${ }^{*}$. By using identities (1)-(8), we can further transform each $\Delta_{j}$ into regular words as we did in Kharchenko's theorem. From now on, unless specified otherwise, whenever we write $\phi\left(x_{i}^{\Delta_{j}},\left(x_{i}^{\Delta_{j}}\right)^{*}\right), \Delta_{j}$ are always understood to be regular words.

Our main objective is to prove the following *-version of Kharchenko's theorem:

Main Theorem. Let $R$ be a prime ring with involution ${ }^{*}$. Suppose that $\phi\left(x_{i}^{\Delta_{j}},\left(x_{i}^{\Delta_{j}}\right)^{*}\right)=0$ is a $*$-differential identity for $R$, where $\Delta_{j}$ are distinct regular words of derivations in $M$ with respect to $<$. Then $\phi\left(z_{i j}, z_{i j}^{*}\right)=0$ is a *-generalized identity for $R$, where $z_{i j}$ are distinct indeterminates.

Now we prove an immediate generalization of Kharchenko's theorem, which will be needed later. Suppose that for each indeterminate $x_{i}$, we pick a basis $M_{0}^{(i)}$ for $D_{\text {int }}$ and then augment $M_{0}^{(i)}$ to a basis $M^{(i)}$ for $D_{\text {int }}+D$. We also fix a total order $<^{(i)}$ in the set $M^{(i)}$ such that $\mu<{ }^{(i)} \mu_{0}$ for $\mu_{0} \in M_{0}^{(i)}$ and $\mu \in M^{(i)} \backslash M_{0}^{(i)}$. We then extend this total order to the set of words in $M^{(i)}$ and also define regular words in $M^{(i)}$ with respect to $<^{(i)}$, as we did before for $M$ with respect to < when we stated Kharchenko's theorem. If desired, 
$M^{(i)},<^{(i)}$ can be made to coincide with each other. But in general, they are completely arbitrary and independent of each other.

By means of the basic identities (1)-(6), any differential identity (without involution $\left.{ }^{*}\right)$ can be brought to the form $\phi\left(x_{i}^{\Delta_{j}^{(i)}}\right)=0$, where

(1) $\phi\left(z_{i j}\right)$ is a generalized polynomial in distinct indeterminates $z_{i j}$, and where

(2) for each $i, \Delta_{j}^{(i)}$ are regular words in $M^{(i)}$.

We have the following:

Generalized Kharchenko theorem. Let $R$ be a prime ring. Suppose that $\phi\left(x_{i}^{\Delta_{j}^{(i)}}\right)=0$ is a differential identity for $R$, where $\Delta_{j}^{(i)}$ are distinct regular words in $M_{i}$ with respect to $<^{(i)}$ for each $i, j$. Then $\phi\left(z_{i j}\right)$ is a generalized identity of $R$, where $z_{i j}$ are distinct indeterminates.

Proof. Let us assign arbitrarily certain fixed values from $R$ to the indeterminates $x_{2}, x_{3}, \ldots$ of $\phi$ other than $x_{1}$. The resulting expression $\phi^{(1)}\left(x^{\Delta_{j}^{(1)}}\right)=0$ is a differential identity involving the indeterminate $x_{1}$ only. Applying the original Kharchenko's theorem to $\phi^{(1)}=0$, we obtain that $\phi^{(1)}\left(z_{1 j}\right)=0$ is a generalized identity for $R$. Since the assignment of values from $R$ to $x_{2}, x_{3}, \ldots$ is completely arbitrary, $\phi\left(z_{1 j}, x_{i}^{\Delta^{(i)}}\right)_{i \geq 2}=0$, the identity obtained by substituting $z_{1 j}$ for $x_{1}^{\Delta_{j}^{(1)}}$ in $\phi\left(x_{i}^{\Delta_{j}^{(i)}}\right)=0$, is still a differential identity for $R$. Continuing this process, we can eventually replace all $x_{i}^{\Delta_{j}^{(i)}}$ by $x_{i j}$. This completes the proof.

If we let $M^{(i)},<^{(i)}$ all coincide with $M=M^{(1)},<=<^{(1)}$, then the above generalized theorem specializes to the original one.

Back to the *-version, let $M_{0}, M,<$ be as explained in Kharchenko's original theorem. Define $M_{0}^{*}=\left\{\mu^{*}: \mu \in M_{0}\right\}$ and $M^{*}=\left\{\mu^{*}: \mu \in M\right\}$. For $\mu_{1}, \mu_{2} \in M$, we define $\mu_{1}^{*}<{ }^{*} \mu_{2}^{*}$ if and only if $\mu_{1}<\mu_{2}$. For a word of derivations $\Delta=\delta_{1} \delta_{2} \cdots \delta_{n}$, where $\delta_{i} \in M$, we define $\Delta^{*}=\delta_{1}^{*} \delta_{2}^{*} \cdots \delta_{n}^{*}$. Note that if $\Delta$ is a regular word in $M$ with respect to $<$, then $\Delta^{*}$ is a regular word in $M^{*}$ with respect to $<^{*}$.

Using the identity $(9)^{\prime}, \phi\left(x_{i}^{\Delta_{j}},\left(x_{i}^{\Delta_{j}}\right)^{*}\right)=0$, where $\Delta_{j}$ are regular words in $M$, can be transformed into $\phi\left(x_{i}^{\Delta_{j}},\left(x_{i}^{*}\right)^{\Delta_{j}^{*}}\right)=0$, where $\Delta_{j}$ and $\Delta_{j}^{*}$ are regular words in $M$ and $M^{*}$, respectively.

\section{SPECIAL $*$-DIFFERENTIAL IDENTITIES}

Let $\psi\left(x_{i}^{\Delta_{j}},\left(x_{i}^{*}\right)^{\Delta_{j}^{\prime}}\right)=0$ be a $*$-differential identity for $R$, where $\Delta_{j}, \Delta_{j}^{\prime}$ are simply words of arbitrary derivations, not necessarily regular words in $M$. Then $\psi\left(x_{i}^{\Delta_{j}},\left(x_{i}^{*}\right)^{\Delta_{j}^{\prime}}\right)=0$ is said to be special for $R$ if $\psi\left(x_{i}^{\Delta_{j}}, y_{i}^{\Delta_{j}^{\prime}}\right)=0$ is a differential identity for $R$, where $y_{i}$ are new indeterminates distinct from 
$x_{i}$. In other words, $\psi\left(x_{i}^{\Delta_{j}},\left(x_{i}^{*}\right)^{\Delta_{j}^{\prime}}\right)=0$ is special for $R$ if $x_{i}$ and $x_{i}^{*}$ can be regarded as independent indeterminates. This generalizes the definition of special generalized identities given in [7, p. 471].

Suppose that we use identities (1)-(6) to transform $\psi\left(x_{i}^{\Delta_{j}},\left(x_{i}^{*}\right)^{\Delta_{j}^{\prime}}\right)=0$ into another identity $\theta\left(x_{i}^{\bar{\Delta}_{j}},\left(x_{i}^{*}\right)^{\bar{\Delta}_{j}^{\prime}}\right)=0$, where $\bar{\Delta}_{j}, \bar{\Delta}_{j}^{\prime}$ are also words of arbitrary derivations (not necessarily regular in $M$ ). Then the same procedure also transforms $\psi\left(x_{i}^{\Delta_{j}}, y_{i}^{\Delta_{j}^{\prime}}\right)=0$ into $\theta\left(x_{i}^{\bar{\Delta}_{j}}, y_{i}^{\bar{\Delta}_{j}^{\prime}}\right)=0$. Thus if $\psi\left(x_{i}^{\Delta_{j}},\left(x_{i}^{*}\right)^{\Delta_{j}}\right)=0$ is special for $R$, then so is $\theta\left(x_{i}^{\bar{\Delta}_{j}},\left(x_{i}^{*}\right)^{\bar{\Delta}_{j}^{\prime}}\right)=0$. We have thus shown that the speciality of $a *$-differential identity is independent of transformations using identities (1)-(6).

If every $*$-differential identity of the form $\psi\left(x_{i}^{\Delta_{j}},\left(x_{i}^{*}\right)^{\Delta_{j}^{\prime}}\right)=0$, where $\Delta_{j}$, $\Delta_{j}^{\prime}$ are words of arbitrary derivations (not necessarily regular), is special for $R$, then $R$ is said to be special. The relationship between the speciality and our Main Theorem is the following:

Lemma 1. If $R$ is special, then the Main Theorem holds.

Proof. Suppose that $\phi\left(x_{i}^{\Delta_{j}},\left(x_{i}^{\Delta_{j}}\right)^{*}\right)=0$ is a $*$-differential identity, where $\Delta_{j}$ are regular words in $M$. Using the identity $(9)^{\prime}, \phi\left(x_{i}^{\Delta_{j}},\left(x_{i}^{\Delta_{j}}\right)^{*}\right)=0$ can be transformed into $\phi\left(x_{i}^{\Delta_{j}},\left(x_{i}^{*}\right)^{\Delta_{j}^{*}}\right)=0$, where $\Delta_{j}^{*}$ are hence regular words in $M^{*}$. Suppose that $R$ is special. Then $\phi\left(x_{i}^{\Delta_{j}}, y_{i}^{\Delta_{j}^{*}}\right)=0$ is a differential identity for $R$, where $y_{i}$ are new indeterminates. By the generalized Kharchenko theorem, $\phi\left(z_{i j}, w_{i j}\right)=0$ is a generalized identity for $R$. In particular, $\phi\left(z_{i j}, z_{i j}^{*}\right)=0$ is a $*$-generalized identity for $R$.

The aim of this section is to prove the following generalization of Theorem 7 [7, p. 473]:

Proposition 1. Suppose that ${ }^{*}$ is of the second kind and that $C$ is infinite. Then $R$ is special.

The proof will be completed by a series of lemmas. First, we recall some more definitions from [3]. Let $Q^{\prime}$ be the ring anti-isomorphic to $Q$ with the same additive group. That is, $Q^{\prime}$ is the opposite of $Q$. Let $B$ denote the subring of the tensor product $Q \otimes_{Z} Q^{\prime}$ (where $Z$ is the ring of integers) generated by the elements of the form $1 \otimes r, r \otimes 1, r \in R$. Elements of $B$ are of the form $\sum_{i} r_{i} \otimes v_{i}$, where $r_{i}, v_{i} \in R$. For $a \in Q, \beta=\sum_{i} r_{i} \otimes v_{i} \in Q \otimes_{Z} Q^{\prime}$, let $a \cdot \beta=\sum_{i} v_{i} a r_{i}$. For $a \in Q$, let $a^{\perp}=\{\beta \in B: a \cdot \beta=0\}$. For $V \subseteq B$, let $V^{\perp}=\left\{a \in R_{F}: a \cdot V=0\right\}$. If $\Delta$ is an endomorphism of the abelian group $Q$, we set $\beta^{\Delta}=\sum_{i} r_{i}^{\Delta} \otimes v_{i}$. (This is well defined!) If $f(x)$ is some linear expression involving the variable $x$ and if $\beta=\sum_{i} r_{i} \otimes v_{i} \in B$, then we set $f(x) \cdot \beta=\sum_{i} v_{i} f\left(r_{i} x\right)$. Observe that if $f(x)$ is identically zero on $R$, then so is $f(x) \cdot \beta$. 
We quote the following [3, Lemma 1]:

Lemma 2. Suppose that $a_{i} \in R_{F}(i=1,2, \ldots, m)$. Then

$$
\sum_{i=1}^{m} a_{i} C=\left(\bigcap_{i=1}^{m} a_{i}^{\perp}\right)^{\perp}
$$

Suppose that $\phi\left(x_{i}^{\Delta_{j}},\left(x_{i}^{\Delta_{j}}\right)^{*}\right)=0$ is a $*$-differential identity, where $\Delta_{j}$ are regular words in $M . \phi\left(x_{i}^{\Delta_{j}},\left(x_{i}^{\Delta_{j}}\right)^{*}\right)=0$ is said to be trivial if the *-generalized polynomial $\phi\left(z_{i j}, z_{i j}^{*}\right)$ is trivial, or equivalently if the ordinary generalized polynomial $\phi\left(z_{i j}, y_{i j}\right)$ (without $\left.{ }^{*}\right)$ is trivial.

A linear *-generalized identity $f(x)$ is of the form

$$
f(x)=\sum_{i} a_{i} x b_{i}+\sum_{j} c_{j} x^{*} d_{j}=0,
$$

where $a_{i}, b_{i}, c_{j}, d_{j} \in R_{F} . f$ is trivial if and only if $\sum_{i} a_{i} \otimes_{C} b_{i}=0$ and $\sum_{j} c_{j} \otimes_{C} d_{j}=0$. A linear *-differential identity $\phi(x)$ can be written in the form

$$
\phi=\phi\left(x^{\Delta_{i}},\left(x^{\Gamma_{j}}\right)^{*}\right)=\sum_{i} \sum_{k=1}^{n_{i}} a_{i}^{(k)} x^{\Delta_{i}} b_{i}^{(k)}+\sum_{j} \sum_{l=1}^{m_{j}} c_{j}^{(l)}\left(x^{\Gamma^{\prime}}\right)^{*} d_{j}^{(l)},
$$

where $a_{i}^{(k)}, b_{i}^{(k)}, c_{j}^{(l)}, d_{j}^{(l)} \in R_{F}$ and where $\Delta_{i}, \Gamma_{j}$ are regular words in $M$. Using the identity $(9)^{\prime}, \phi$ can also be written in the form

$$
\phi=\phi\left(x^{\Delta_{i}},\left(x^{*}\right)^{\Gamma_{j}^{*}}\right)=\sum_{i} \sum_{k=1}^{n_{i}} a_{i}^{(k)} x^{\Delta_{i}} b_{i}^{(k)}+\sum_{j} \sum_{l=1}^{m_{j}} c_{j}^{(l)}\left(x^{*}\right)^{\Gamma_{j}^{*}} d_{j}^{(l)},
$$

where $\Gamma_{j}^{*}$ are, certainly, regular words in $M^{*}$. $\phi$ is said to be trivial if and only if $\sum_{k=1}^{n_{1}} a_{i}^{(k)} \otimes_{C} b_{i}^{(k)}=0$ for all $i$ and $\sum_{l=1}^{m_{J}} c_{j}^{(l)} \otimes_{C} d_{j}^{(l)}=0$ for all $j$.

The following lemma is essentially the *-version of Lemma 2 [3].

Lemma 3. Suppose that every linear *-generalized identity of $R$ is trivial. Then so is every linear *-differential identity of $R$.

Proof. We need the following formula from [3, p. 158]: Let $\Delta=\delta_{1} \cdots \delta_{m}$ be a regular word in $M$ such that $\delta_{1}=\delta_{2}=\cdots=\delta_{s} \neq \delta_{s+1}$, where $0<s<p$ if ch $R=p>0$. Let $\beta \in B$. Then

$$
\left(a x^{\Delta} b\right) \cdot \beta=(a \cdot \beta) x^{\Delta} b+s\left(a \cdot \beta^{\delta_{1}}\right) x^{\delta_{2} \cdots \delta_{m}} b+\cdots,
$$

where the dots above denote a sum of terms $d x^{\Delta^{\prime}} b$ in which $\Delta^{\prime}<\delta_{2} \cdots \delta_{m}$.

We define a partial order on ordered pairs of regular words in $M$. Assume that $\Delta_{1}, \Delta_{2}, \Gamma_{1}$, and $\Gamma_{2}$ are regular words in $M$. We define $\left(\Delta_{1}, \Gamma_{1}\right)>$ $\left(\Delta_{2}, \Gamma_{2}\right)$ if $\dot{\Delta}_{1}>\Delta_{2}$ and $\Gamma_{1} \geq \Gamma_{2}$ or if $\Delta_{1} \geq \Delta_{2}$ and $\Gamma_{1}>\Gamma_{2}$.

Let $\bar{M}$ be a finite subset of $M$. Note that the set of all regular words in $\bar{M}$ is well ordered under $<$. Hence the partial order $<$ on word pairs defined 
above is well-founded when restricted to ordered pairs of regular words in $\bar{M}$. Let $\mathscr{I}(\bar{M})$ denote the set of all linear $*$-differential identities involving only derivation words in $\bar{M} . \mathscr{I}(\bar{M})$ consists of the $*$-differential identities of the form

$$
\sum_{i} \sum_{k} A_{i k} x^{\bar{\Delta}_{i}} B_{i k}+\sum_{j} \sum_{l} C_{j l}\left(x^{\bar{\Gamma}_{j}}\right)^{*} D_{j l}=0
$$

where $\bar{\Delta}_{i}, \bar{\Gamma}_{j}$ are regular words in $\bar{M}$.

Now suppose that

$$
\phi(x)=\sum_{i} \sum_{k=1}^{n_{i}} a_{i}^{(k)} x^{\Delta_{i}} b_{i}^{(k)}+\sum_{j} \sum_{l=1}^{m_{j}} c_{j}^{(l)}\left(x^{\Gamma_{j}}\right)^{*} d_{j}^{(l)}=0
$$

is an arbitrary linear *-differential identity in $\mathscr{I}(\bar{M})$; that is, $\Delta_{i}, \Gamma_{j}$ are regular words in $\bar{M}$. We may assume that $\left(\Delta_{1}, \Gamma_{1}\right)$ is the leading word pair of $\phi$; that is, $\Delta_{1} \geq \Delta_{i}$ and $\Gamma_{1} \geq \Gamma_{j}$ for all $i, j$. We proceed by induction on the leading word pair $\left(\Delta_{1}, \Gamma_{1}\right)$ (under the partial order defined on word pairs) to show that $\phi$ is trivial.

If $\left(\Delta_{1}, \Gamma_{1}\right)=(\varnothing, \varnothing)$, then $\phi$ is simply an ordinary $*$-generalized identity without derivations. By our assumption, $\phi$ must be trivial. So suppose that $\left(\Delta_{1}, \Gamma_{1}\right) \neq(\varnothing, \varnothing)$. Let us first assume that $\Delta_{1} \neq \varnothing$. Obviously $a_{1}^{(1)}, \ldots, a_{1}^{\left(n_{1}\right)}$ can be assumed to be $C$-linearly independent.

We claim that it suffices to prove the case when $n_{1}=1$ : Suppose that $n_{1}>1$ is given. Let $\beta \in \bigcap_{k \geq 2}^{n_{1}}\left(a_{1}^{(k)}\right)^{\perp}$. Using formula (1), $\phi(x) \cdot \beta$ has a single term $\left(a_{1}^{(1)} \cdot \beta\right) x^{\Delta_{1}} b_{1}^{(1)}$ containing the regular word $\Delta_{1}$. If we have proved the case when $n_{1}=1$, then $\phi(x) \cdot \beta$ is trivial and hence $a_{1}^{(1)} \cdot \beta=0$. So $a_{1}^{(1)} \cdot \beta=0$ for all $\beta \in \bigcap_{k \geq 2}^{n_{1}}\left(a_{1}^{(k)}\right)^{\perp}$ and, by Lemma 2, $a_{1}^{(1)} \in \sum_{k \geq 2}^{n_{1}} a_{1}^{(k)} C$, a contradiction to the $C$-linear independence of $a_{1}^{(1)}, \ldots, a_{1}^{\left(n_{1}\right)}$.

So we may assume $n_{1}=1$. Suppose $\Delta_{1}=\delta_{1} \delta_{2} \cdots \delta_{m}$, where $\delta_{1}=\delta_{2}=\cdots=$ $\delta_{s} \neq \delta_{s+1}$ and $0<s<p$ if $\operatorname{ch} R=p>0$. Set $\bar{\Delta}_{1}=\delta_{2} \cdots \delta_{m}$. Let $\mu_{1}, \ldots, \mu_{n}$ be all derivations in $\bar{M}$ other than $\delta_{1}$ such that $\mu_{1} \bar{\Delta}_{1}, \ldots, \mu_{n} \bar{\Delta}_{1}$ occur in $\phi$ as some $\Delta_{i}$. Let us say $\Delta_{r_{1}}=\mu_{1} \bar{\Delta}_{1}, \ldots, \Delta_{r_{n}}=\mu_{n} \bar{\Delta}_{1}$. We also assume that $\bar{\Delta}_{1}$ occurs in $\phi$ as $\Delta_{k_{0}}$. In view of formula (1), the sum of terms of $\phi(x) \cdot \beta$ containing $x^{\bar{\Delta}_{1}}$ has the following form:

$$
s\left(a_{1}^{(1)} \cdot \beta^{\delta_{1}}\right) x^{\bar{\Delta}_{1}} b_{1}^{(1)}+\sum_{\tau=1}^{n} \sum_{k=1}^{n_{r_{\tau}}}\left(a_{r_{\tau}}^{(k)} \cdot \beta^{\mu_{\tau}}\right) x^{\bar{\Delta}_{1}} b_{r_{\tau}}^{(k)}+\sum_{i}\left(a_{k_{0}}^{(i)} \cdot \beta\right) x^{\bar{\Delta}_{1}} b_{k_{0}}^{(i)} .
$$

If $a_{1}^{(1)} \cdot \beta=0$, then the leading word pair of $\phi(x) \cdot \beta$ is strictly less than that of $\phi$. Since $\phi(x) \cdot \beta \in \mathscr{I}(\bar{M})$, by our induction hypothesis, $\phi(x) \cdot \beta$ must be trivial. So we have

$$
s\left(a_{1}^{(1)} \cdot \beta^{\delta_{1}}\right) \otimes_{C} b_{1}^{(1)}+\sum_{\tau, k}\left(a_{r_{\tau}}^{(k)} \cdot \beta^{\mu_{\tau}}\right) \otimes_{C} b_{r_{\tau}}^{(k)}+\sum_{i}\left(a_{k_{0}}^{(i)} \cdot \beta\right) \otimes_{C} \cdot b_{k_{0}}^{(i)}=0 .
$$


Since $0<s<p$ in the case of $\operatorname{ch} R=p>0, s$ is nonzero as an element of $C$. Hence $a_{1}^{(1)} \cdot \beta^{\delta_{1}}$ can be linearly expressed in terms of $a_{r_{\tau}}^{(k)} \cdot \beta^{\mu_{\tau}}$ and $a_{k_{0}}^{(i)} \cdot \beta$. Using the linearity of ()$\cdot \beta^{\mu_{\tau}},() \cdot \beta$, and introducing new notation, we have

$$
a_{1}^{(1)} \cdot \beta^{\delta_{1}}+\sum_{\tau} d_{\tau} \cdot \beta^{\mu_{\tau}}+h \cdot \beta=0 .
$$

Since the right coefficients of (2) do not depend on $\beta$, neither do $d_{\tau}$ and $h$. This shows that the mapping

$$
\zeta: a_{1}^{(1)} \cdot \beta \mapsto a_{1}^{(1)} \cdot \beta^{\delta_{1}}+\sum_{\tau} d_{\tau} \cdot \beta^{\mu_{\tau}}+h \cdot \beta,
$$

where $\beta$ ranges over $B$, is well defined. Choose $I \in F$ such that $I a_{1}^{(1)} \subseteq R$, $I d_{\tau} \subseteq R \quad(\tau=1, \ldots, n)$, and $I h \subseteq R$. Set $T=B(1 \otimes I)$. If $\beta \in T=B(1 \otimes I)$, then both $a_{1}^{(1)} \cdot \beta$ and $\zeta\left(a_{1}^{(1)} \cdot \beta\right)$ fall in $R$. Thus $\zeta$ is defined on the ideal $\left\{a_{1}^{(1)} \cdot \beta: \beta \in T\right\}$ of $R$ and the range of this ideal under $\zeta$ is also contained in $R$. Furthermore, for $v \in R$,

$$
\begin{aligned}
\zeta\left(v\left(a_{1}^{(1)} \cdot \beta\right)\right) & =\zeta\left(a_{1}^{(1)} \cdot \beta(1 \otimes v)\right) \\
& =a_{1}^{(1)} \cdot \beta^{\delta_{1}}(1 \otimes v)+\sum_{\tau} d_{\tau} \cdot \beta^{\mu_{\tau}}(1 \otimes v)+h \cdot \beta(1 \otimes v) \\
& =v \zeta\left(a_{1}^{(1)} \cdot \beta\right) .
\end{aligned}
$$

Hence, by the definition of $R_{F}$, there exists $t \in R_{F}$ such that, for $\beta \in T$,

$$
\left(a_{1}^{(1)} \cdot \beta\right) t=\zeta\left(a_{1}^{(1)} \cdot \beta\right)=a_{1}^{(1)} \cdot \beta^{\delta_{1}}+\sum_{\tau} d_{\tau} \cdot \beta^{\mu_{\tau}}+h \cdot \beta .
$$

For $x \in R$, we compute

$$
\begin{aligned}
\left(a_{1}^{(1)} \cdot \beta\right)(x t)= & \left(\left(a_{1}^{(1)} \cdot \beta\right) x\right) t=\left(a_{1}^{(1)} \cdot \beta(x \otimes 1)\right) t \\
= & a_{1}^{(1)} \cdot(\beta(x \otimes 1))^{\delta_{1}}+\sum_{\tau} d_{\tau} \cdot(\beta(x \otimes 1))^{\mu_{\tau}}+h \cdot(\beta(x \otimes 1)) \\
= & \left(a_{1}^{(1)} \cdot \beta^{\delta_{1}}\right) x+\left(a_{1}^{(1)} \cdot \beta\right) x^{\delta_{1}} \\
& +\sum_{\tau}\left(d_{\tau} \cdot \beta^{\mu_{\tau}}\right) x+\sum_{\tau}\left(d_{\tau} \cdot \beta\right) x^{\mu_{\tau}}+(h \cdot \beta) x
\end{aligned}
$$

and

$$
\left(a_{1}^{(1)} \cdot \beta\right)(t x)=\left(\left(a_{1}^{(1)} \cdot \beta\right) t\right) x=\left(a_{1}^{(1)} \cdot \beta^{\delta_{1}}\right) x+\sum_{\tau}\left(d_{\tau} \cdot \beta^{\mu_{\tau}}\right) x+(h \cdot \beta) x .
$$

Hence we obtain

$$
\left(a_{1}^{(1)} \cdot \beta\right)[x, t]=\left(a_{1}^{(1)} \cdot \beta\right)(x t-t x)=\left(a_{1}^{(1)} \cdot \beta\right) x^{\delta_{1}}+\sum_{\tau}\left(d_{\tau} \cdot \beta\right) x^{\mu_{\tau}} .
$$

Now suppose that $a_{1}^{(1)}=a_{1}, a_{2}, \ldots$ is a basis for the subspace $a_{1}^{(1)} \cdot C+$ $\sum_{\tau} d_{\tau} \cdot C$. Express $d_{\tau}$ in terms of this basis:

$$
d_{\tau}=\alpha_{\tau} a_{1}+\cdots, \quad \alpha_{\tau} \in C .
$$


Let $\beta \in \bigcap_{i \geq 2} a_{i}^{\perp} \cap T$. Then $d_{\tau} \cdot \beta=\alpha_{\tau}\left(a_{1} \cdot \beta\right)$. By (3), we have

$$
\left(a_{1}^{(1)} \cdot \beta\right)\left([x, t]-x^{\delta_{1}}-\sum_{\tau} \alpha_{\tau} x^{\mu_{\tau}}\right)=0 .
$$

Hence $[x, t]=x^{\delta_{1}}+\sum_{\tau} \alpha_{\tau} x^{\mu_{\tau}}$. This contradicts with our choice of the basis $M$. So we must assume that $\Delta_{1}=\varnothing$ and $\Gamma_{1} \neq \varnothing$.

Replacing $x$ by $x^{*}$ in $\phi$ and using the identity $(9)^{\prime}$ to bring ${ }^{*}$ inside of derivations, we have

$$
\phi\left(x^{*}\right)=\sum_{i} \sum_{k=1}^{n_{i}} a_{i}^{(k)}\left(x^{*}\right)^{\Delta_{i}} b_{i}^{(k)}+\sum_{j} \sum_{l=1}^{m_{j}} c_{j}^{(l)} x^{\left(\Gamma_{j}^{*}\right)} d_{j}^{(l)} .
$$

By a similar induction on regular words in $\bar{M}^{*}=\left\{\delta^{*}: \delta \in \bar{M}\right\}$ with respect to $<^{(*)}$, we can show that $\Gamma_{j}^{*}=\varnothing$. This is another contradiction. We have thus shown that any linear $*$-differential identity of $\mathscr{I}(\bar{M})$ is trivial.

Now let

$$
\phi(x)=\sum_{i} \sum_{k=1}^{n_{i}} a_{i}^{(k)} x^{\Delta_{i}} b_{i}^{(k)}+\sum_{j} \sum_{l=1}^{m_{j}} c_{j}^{(l)}\left(x^{\Gamma_{j}}\right)^{*} d_{j}^{(l)}=0
$$

be an arbitrary linear $*$-differential identity. Let $\bar{M}$ be the finite subset of $M$ consisting of all derivations occurring in $\Delta_{i}$ and $\Gamma_{j}$. Then $\phi \in \mathscr{I}(\bar{M})$. By the result of the previous paragraph, $\phi$ must be trivial.

Lemma 4. Suppose that every linear *-generalized identity of $R$ is trivial. If $\phi\left(x_{i}^{\Delta_{j}},\left(x_{i}^{\Delta_{j}}\right)^{*}\right)=0$, where $\Delta_{j}$ are distinct regular words in $M$, is a multilinear *-differential identity for $R$, then $\phi\left(z_{i j}, y_{i j}\right)=0$, where $z_{i j}, y_{i j}$ are distinct indeterminates, is a multilinear generalized identity of $R$.

Proof. If $\phi$ involves only one indeterminate, say $x_{1}$, then $\phi=\phi\left(x_{1}^{\Delta_{j}},\left(x_{1}^{\Delta_{j}}\right)^{*}\right)$ is trivial by Lemma 3 . Hence $\phi\left(z_{1 j}, y_{1 j}\right)=0$ is also a trivial generalized identity for $R$.

Now suppose that $\phi$ involves more than one indeterminates, say $x_{1}, \ldots, x_{n}$. Let us assign certain fixed values from $R$ to $x_{2}, \ldots, x_{n}$. The resulting identity thus involves the indeterminate $x_{1}$ only and hence must be trivial by Lemma 3 . As in the previous paragraph, we can replace $x_{1}^{\Delta_{j}},\left(x_{1}^{\Delta_{j}}\right)^{*}$ by new indeterminates $z_{1 j}, y_{1 j}$ to obtain a generalized identity. Since the values assigned to $x_{2}, \ldots, x_{n}$ are completely arbitrary, $\phi\left(z_{1 j}, y_{1 j}, x_{i}^{\Delta_{j}},\left(x_{i}^{\Delta_{j}}\right)^{*}\right)_{i \geq 2}=0$, the identity obtained by replacing $x_{1}^{\Delta_{j}}, y_{1}^{\Delta_{j}}$ in $\phi=0$ by $z_{1 j}, y_{1 j}$, respectively, also holds on $R$. Repeating the same argument for $x_{2}, \ldots, x_{n}$, the desired result follows.

By assigning fixed values to all indeterminates but one, we can reduce a given identity to an identity involving only one indeterminate. This technique, used in proving the generalized Kharchenko theorem and also in the proof above, will be used frequently throughout this paper. 
Lemma 5. Suppose that ${ }^{*}$ is of the second kind. Then every multilinear *-differential identity of $R$ is special.

Proof. By Theorem 7 [8, p. 473], every multilinear $*$-generalized identity of $R$ is special. In particular, every linear $*$-generalized identity is special and hence must be trivial by Lemma 1.3.2 [1, p. 22]. The result now follows from Lemma 4.

Lemma 6. Suppose that ${ }^{*}$ is of the second kind and that $\operatorname{ch} R=0$. Then $R$ is special.

Proof. Let $\phi$ be a given $*$-differential identity. Replacing each indeterminate $x_{i}$ by $2 x_{i}, 3 x_{i}, \ldots$ and using the Vandermonde determinant, we can solve the homogeneous components of $\phi$. Hence each homogeneous component of $\phi$ is also a $*$-differential identity of $R$. It suffices to show that each homogeneous component of $\phi$ is special. Replacing $\phi$ by one of its homogeneous components, we may assume from the start that $\phi$ is homogeneous in each indeterminate.

Let $\theta$ be the multilinearization of $\phi$. By Lemma $5, \theta$ is special. Identifying the indeterminates in $\theta$ properly, we obtain $n \phi$, where $n$ is a nonzero integer. Hence $n \phi$ is also special and so is $\phi$, since $\operatorname{ch} R=0$.

Lemma 7. Suppose that ${ }^{*}$ is of the second kind and that $\operatorname{ch} R=p>0$. If $C$ is infinite, then $R$ is special.

Proof. By the technique used in proving Lemma 4 (and also the remark following), it suffices to prove the case when $\phi$ involves only one indeterminate, say $x$. We proceed by induction on the degree of $\phi$. If $\operatorname{deg} \phi=1$, then $\phi$ is linear. The result follows from Lemma 5. So we assume that $\operatorname{deg} \phi>1$. As the induction hypothesis, we also assume that the result holds for any $*$-differential identity with less degree. Bringing ${ }^{*}$ inside of derivations by identity $(9)^{\prime}$ and suppressing words of derivations for simplicity of notation, we write $\phi\left(x, x^{*}\right)$ for $\phi\left(x^{\Delta_{j}},\left(x^{*}\right)^{\Delta_{j}^{*}}\right)$. Now consider

$$
\theta\left(x, x^{*}, y, y^{*}\right)=\phi\left(x+y, x^{*}+y^{*}\right)-\phi\left(x, x^{*}\right)-\phi\left(y, y^{*}\right) .
$$

By the induction hypothesis (and the remark following Lemma 4), $\theta$ is special for $R$. Now regard $x, x^{*}, y$, and $y^{*}$ in $\theta$ as independent indeterminates as granted. Set $x=x, x^{*}=0, y=0$, and $y^{*}=x^{*}$ in $\theta$ :

$$
\theta\left(x, 0,0, x^{*}\right)=\phi\left(x, x^{*}\right)-\phi(x, 0)-\phi\left(0, x^{*}\right) .
$$

Thus $\phi(x, 0)+\phi\left(0, x^{*}\right)$ is also a $*$-differential identity for $R$. We also have the identity $\phi\left(x, x^{*}\right)=\theta\left(x, 0,0, x^{*}\right)+\phi(x, 0)+\phi\left(0, x^{*}\right)$. Since $\theta\left(x, 0,0, x^{*}\right)$ is special for $R$, it suffices to prove that $\phi(x, 0)+\phi\left(0, x^{*}\right)$ is special. This is equivalent to showing that both $\phi(x, 0)=0$ and $\phi\left(0, x^{*}\right)=0$ are differential identities for $R$.

Set $\zeta(x)=\phi(x, 0)$ and $\eta\left(x^{*}\right)=\phi\left(0, x^{*}\right)$. For positive integers $t \geq 0$, let $\zeta_{t}(x)$ and $\eta_{t}\left(x^{*}\right)$ denote the sums of those monomials with degree $t$ in $\zeta(x)$ 
and in $\eta\left(x^{*}\right)$, respectively. Pick sufficiently but finitely many $\alpha \in C$ such that $\alpha^{*}=\alpha$. Let $I$ be a nonzero $*$-ideal of $R$ such that $\alpha^{p} I \subseteq R$ for those finitely many $\alpha \in C$ we have picked. Then $\zeta\left(\alpha^{p} x\right)+\eta\left(\alpha^{p} x^{*}\right)$ vanishes on $I$. Since $\alpha^{p}$ are constants of any derivations, we can use the Vandermonde determinant to solve the homogeneous components of $\zeta\left(\alpha^{p} x\right)+\eta\left(\alpha^{p} x^{*}\right)=0$. So we have that, for each $t \geq 0, \zeta_{t}(x)+\eta_{t}\left(x^{*}\right)$ vanishes on $I$.

We claim that there exists $\alpha \in C$ such that $\alpha^{p t} \neq\left(\alpha^{*}\right)^{p t}$ : Pick $\beta \in C$ such that $\beta^{*} \neq \beta$. For $s \in C$ such that $s^{*}=s$, set $\alpha(s)=s+\beta$. Observe that

$$
\frac{\alpha(s)^{*}}{\alpha(s)}=\frac{s+\beta^{*}}{s+\beta}=1+\frac{\beta^{*}-\beta}{s+\beta} \text {. }
$$

Hence if $s \neq s^{\prime}$, then $\alpha(s)^{*} / \alpha(s) \neq \alpha\left(s^{\prime}\right)^{*} / \alpha\left(s^{\prime}\right)$. Since the equation $y^{p t}=1$ has only finitely many solutions in the infinite field $C$, there exists a symmetric element $s \in C$ such that $\alpha(s)^{p t} \neq\left(\alpha(s)^{*}\right)^{p t}$.

Pick $\alpha \in C$ such that $\alpha^{p t} \neq\left(\alpha^{*}\right)^{p t}$ as claimed above. Let $J$ be a nonzero *-ideal of $R$ such that $\alpha^{P} J \subseteq R$. Then $\zeta_{t}\left(\alpha^{p} x\right)+\eta_{t}\left(\left(\alpha^{p} x\right)^{*}\right)=0$ holds for $x \in I J$. Hence for $x \in I J, 0=\alpha^{p t}\left(\zeta_{t}(x)+\eta_{t}\left(x^{*}\right)\right)-\left(\zeta_{t}\left(\alpha^{p} x\right)+\eta_{t}\left(\left(\alpha^{p} x\right)^{*}\right)\right)=$ $\left(\alpha^{p t}-\left(\alpha^{*}\right)^{p t}\right) \eta_{t}\left(x^{*}\right)$. Thus $\eta_{t}\left(x^{*}\right)=0$ holds on $I J$. Similarly, $\zeta_{t}(x)=0$ also holds on $I J$. Note that $\zeta_{t}(x)=0$ and $\eta_{t}(x)=0$ are simply ordinary differential identities without the involution ${ }^{*}$. By the remark on p. 74 in [4], $\zeta_{t}(x)=0$ and $\eta_{t}(x)=0$ also hold on $R$. This completes the proof.

Proposition 1 now follows from Lemma 6 and Lemma 7.

One might wonder whether or not the assumption that $C$ is infinite can be eliminated in Proposition 1. The answer is negative, as the following example shows.

Example. Fix a positive integer $n \geq 1$. Let $\Phi$ be a finite field of characteristic $p>0$ with $p^{2 n}$ elements. For $\alpha \in \Phi$, define $\bar{\alpha}=\alpha^{p^{n}}$. - is an involution of the second kind on $\Phi$. The symmetric elements of $\Phi$ satisfy the polynomial identity $s^{p^{n}}=s$. But the $*$-identity $(x+\bar{x})^{p^{n}}=x+\bar{x}$ is obviously not special.

For a noncommutative example, we can take any $k \times k$ matrix ring $\Phi_{k}$ $(k \geq 1)$ with transpose involution ${ }^{*}$ defined as follows: For $\alpha_{i j} \in \Phi(i, j=$ $1, \ldots, k),\left(\alpha_{i j}\right)^{*}=\left(\bar{\alpha}_{j i}\right)$. For an infinite-dimensional example, let $V$ be an infinite-dimensional vector space over $\Phi$. Pick a Hermitian bilinear form ( , ) on ${ }_{\Phi} V$. That is, ( , ) satisfies the condition $(\alpha v, \beta w)=\alpha \bar{\beta}(v, w)$ for $\alpha, \beta \in$ $\Phi$ and $v, w \in V$. Our desired ring is the set consisting of all continuous finite rank linear maps in $\operatorname{End}\left({ }_{\Phi} V\right)$. (See $\S I I I$ for definitions.)

Actually, we can prove that any counterexample to Proposition 1 must be of the form described above.

\section{III. *-RINGS HAVING MINIMAL ONE-SIDED IDEALS}

We digress here to generalize some basic theorems on rings having minimal one-sided ideals to their $*$-versions. 
First, we recall some basic definitions. Let $D$ be a division ring. Suppose that ${ }_{D} V$ is a left vector space over $D$ and $W_{D}$, a right vector space over $D$. If there exists a nondegenerate bilinear form $():, V \times W \rightarrow D$, then ${ }_{D} V$ and $W_{D}$ are said to be dual with respect to $($,$) .$

Let $A(V)=\operatorname{Hom}\left({ }_{D} V,{ }_{D} V\right)$ and $A(W)=\operatorname{Hom}\left(W_{D}, W_{D}\right)$. For $a \in A(V)$ and $b \in A(W)$, if $(v a, w)=(v, b w)$ holds for all $v \in V$ and for all $w \in W$, then we call $b$ an adjoint of $a$. Not every element $a \in A(V)$ has an adjoint. But if $a \in A(V)$ does have an adjoint, then that adjoint must be unique and will be denoted by $a^{*}$. $a \in A(V)$ is said to be continuous if $a$ has an adjoint $a^{*} \in A(W)$. The set of all continuous elements $a \in A(V)$ forms a ring and is denoted by $L_{W}(V)$. Let $S_{W}(V)=\left\{a \in L_{W}(V): \operatorname{dim}_{D}(V a)<\infty\right\}$. For $v_{1}, \ldots, v_{n} \in V$ and $w_{1}, \ldots, w_{n} \in W$, the linear transformation $a \in A(V)$ defined by $v a=\left(v, w_{1}\right) v_{1}+\cdots+\left(v, w_{n}\right) v_{n}$ is in $S_{W}(V)$ and has the adjoint $a^{*} \in S_{V}(W)$ given by $a^{*} w=w_{1}\left(v_{1}, w\right)+\cdots+w_{n}\left(v_{n}, w\right)$. Conversely, any $a \in S_{W}(V)$ can be represented in the form described above. The following two basic facts can be found in [2].

Fact 1. Let $v_{1}, \ldots, v_{n} \in V$ be $D$-independent. Then there exist $D$-independent vectors $u_{1}, \ldots, u_{n} \in W$ such that $\left(v_{i}, u_{j}\right)=\delta_{i j}$ for $i, j=1,2, \ldots, n$.

Fact 2. Let $V_{0}$ and $W_{0}$ be finite-dimensional subspaces of ${ }_{D} V$ and $W_{D}$, respectively. Then there exist finite-dimensional subspaces $V_{1} \supseteq V_{0}$ and $W_{1} \supseteq W_{0}$ of ${ }_{D} V$ and $W_{D}$ respectively such that the bilinear form $($,$) restricted to V_{1} \times W_{1}$ is nondegenerate. (Hence $\operatorname{dim}_{D} V_{1}=\operatorname{dim} W_{1 D}$ by Fact 1.)

For a prime ring $R$, let $\operatorname{Soc}(R)$ be the sum of all minimal right ideals of $R$. It is well known that $\operatorname{Soc}(R)$ is also the sum of all minimal left ideals of $R$ and that $\operatorname{Soc}(R)$, if nonzero, is the unique minimal ideal of $R$. We have the following representation theorem for prime rings with nonzero socle.

Jacobson theorem. Let ${ }_{D} V$ and $W_{D}$ be dual spaces over a division ring $D$ with respect to the nondegenerate bilinear form ( , ). Let $R$ be a subring of $A(V)$ such that $S_{W^{\prime}}(V) \subseteq R \subseteq L_{W^{\prime}}(V)$. Then $R$ is a primitive ring with $\operatorname{Soc}(R)=$ $S_{W^{\prime}}(V)$.

Conversely, given a prime ring $R$ with $\operatorname{Soc}(R) \neq 0$, we can find a division ring $D$ and a pair of dual spaces ${ }_{D} V$ and $W_{D}$ such that $S_{W^{*}}(V) \subseteq R \subseteq L_{W^{*}}(V)$.

The following fact can be found in [4, p. 171] or Proposition 7, [6, p. 98]. However, we give here a new proof using the density theorem, which is more related to our next proposition.

Fact 3. Suppose that $S_{W}(V) \subseteq R \subseteq L_{U} \cdot(V)$. Then $R_{F}=A(V)$.

Proof. Let $h \in R_{F}$. Since $S_{W^{\prime}}(V)$ is the unique minimal ideal of $R$, $S_{W^{\prime}}(V) h \subseteq R$. We define $\tilde{h} \in A(V)$ as follows: For $v_{i} \in V$ and $r_{i} \in S_{W^{*}}(V)$, $\tilde{h}: \sum v_{i} r_{i} \mapsto \sum v_{i}\left(r_{i} h\right)$. Note that $r_{i} h \in R$ and hence $v_{i}\left(r_{i} h\right) \in V$. We must show that $\tilde{h}$ is well defined: Suppose $\sum v_{i} r_{i}=0$. Since $r_{i} \in S_{W^{\prime}}(V)$, 
$\operatorname{dim}_{D}\left(\sum_{i} V r_{i}\right)<\infty$. Choose $r \in S_{W}(V)$ such that $r$ restricted to $\sum_{i} V r_{i}$ is the identity map. Then $r_{i} r=r_{i}$. Hence

$$
\sum v_{i}\left(r_{i} h\right)=\sum v_{i}\left(\left(r_{i} r\right) h\right)=\sum v_{i}\left(r_{i}(r h)\right)=\left(\sum v_{i} r_{i}\right)(r h)=0 .
$$

Via this natural embedding $h \rightarrow \tilde{h}$, we have $R_{F} \subseteq A(V)$.

Conversely, suppose that $h \in A(V)$. In order to prove that $h \in R_{F}$, we must show that $S_{W}(V) h \subseteq R$ : Every element $a \in S_{W}(V)$ can be represented by the form $v a=\sum_{i}\left(v, w_{i}\right) v_{i}$, where $v, v_{i} \in V, w_{i} \in W$. Hence vah= $\sum_{i}\left(v, w_{i}\right)\left(v_{i} h\right)$. Then $a h \in S_{W}(V) \in R$ as desired.

The following proposition is interesting in itself.

Proposition 2. Suppose that $S_{W}(V) \subseteq R \subseteq L_{W}(V)$. Then $Q=L_{W}(V)$, where $Q$ is the two-sided quotient ring of $R$.

Proof. Suppose that $h \in L_{W}(V)$. Then $h S_{W}(V) \subseteq S_{W}(V) \subseteq R$ and $S_{W}(V) h \subseteq$ $S_{W}(V) \subseteq R$. So $h \in Q$.

Conversely, since $Q \subseteq R_{F}$, by Fact 3 above, we may assume $Q \subseteq A(V)$. Let $h \in Q$. In order to show that $h \in L_{W}(V)$, we must find its adjoint $h^{*} \in A(W)$.

Let $S_{W}(V)^{*}=\left\{r^{*}: r \in S_{W}(V)\right\}, R^{*}=\left\{r^{*}: r \in R\right\}$, and $L_{W}(V)^{*}=\left\{r^{*}: r \in\right.$ $\left.L_{W}(V)\right\}$. Observe that $S_{W}(V)^{*}=S_{V}(W)$ and $L_{W}(V)^{*}=L_{V}(W)$. Hence $S_{V}(W) \subseteq R^{*} \subseteq L_{V}(W)$. Let $F^{*}$ be the filter of nonzero two-sided ideals of $R^{*}$ and let ${ }_{F^{*}} R^{*}$ be the right quotient ring of $R^{*}$ relative to the filter $F^{*}$. By a symmetrical version of Fact 3 for right quotient rings, we have ${ }_{F^{*}} R^{*}=A(W)$.

Given $h \in Q$, we define $h^{*}: S_{V}(W) \rightarrow R^{*}$ as follows: For $t \in S_{W}(V)$, set $h^{*}\left(t^{*}\right)=(h t)^{*}$. Since $h \in Q$, we have $h S_{W}(V) \subseteq R$ as well as $S_{W}(V) h \subseteq R$. So $h t \in R$ and hence $(h t)^{*}$ exists. Thus $h^{*}$ is well defined.

For $r \in R$ and $t \in S_{W}(V), h^{*}\left(t^{*} r^{*}\right)=h^{*}\left((t r)^{*}\right)=(h(t r))^{*}=((h t) r)^{*}=$ $(h t)^{*} r^{*}=h^{*}\left(t^{*}\right) r^{*}$. So $h^{*}$ is a right $R^{*}$-homomorphism and hence $h^{*} \in_{F^{*}} R^{*}=$ $A(W)$. To prove that $h^{*}$ is the adjoint of $h$, we must verify that $\left(v, h^{*} w\right)=$ $(v h, w)$ for any $v \in V, w \in W$.

Using the fact that $S_{V}(W)=S_{W}(V)^{*}$ and the Jacobson density theorem, there exists $t \in S_{W}(V)$ such that $t^{*} w=w$. Then we compute

$$
\begin{aligned}
\left(v, h^{*} w\right) & =\left(v, h^{*}\left(t^{*} w\right)\right)=\left(v,\left(h^{*} t^{*}\right) w\right)=\left(v,(h t)^{*} w\right) \\
& =(v(h t), w)=((v h) t, w)=\left(v h, t^{*} w\right)=(v h, w) .
\end{aligned}
$$

So $h^{*}$ is really the adjoint of $h$ and hence $h \in L_{W}(V)$ as desired.

The following generalization of Theorem 7.3.16 [9, p. 269] is the $*$-version of the Jacobson density theorem.

Proposition 3. Suppose that ${ }_{D} V$ and $W_{D}$ are dual spaces with respect to the nondegenerate bilinear form $($,$) . Let v_{1}, \ldots, v_{s}, v_{1}^{\prime}, \ldots, v_{s}^{\prime} \in V$, and $u_{1}, \ldots, u_{t}, u_{1}^{\prime}, \ldots, u_{t}^{\prime} \in W$ be such that $\left\{v_{1}, \ldots, v_{s}\right\}$ is D-independent in $V$ and $\left\{u_{1}, \ldots, u_{t}\right\}$ is D-independent in $W$. Then there exists $a \in S_{W}(V)$ 
such that $v_{i} a=v_{i}^{\prime}(i=1, \ldots, s)$ and $a^{*} u_{j}=u_{j}^{\prime}(j=1, \ldots, t)$ if and only if $\left(v_{i}^{\prime}, u_{j}\right)=\left(v_{i}, u_{j}^{\prime}\right)$ for $i=1, \ldots, s$ and $j=1, \ldots, t$.

Proof. The necessity is easy: Suppose that there exists $a \in S_{W}(V)$ such that $v_{i} a=v_{i}^{\prime}(i=1, \ldots, s)$ and $a^{*} u_{j}=u_{j}^{\prime} \quad(j=1, \ldots, t)$. Then

$$
\left(v_{i}^{\prime}, u_{j}\right)=\left(v_{i} a, u_{j}\right)=\left(v_{i}, a^{*} u_{j}\right)=\left(v_{i}, u_{j}^{\prime}\right) .
$$

To prove the sufficiency, by Fact 2, there exist finite-dimensional subspaces $V_{0}, W_{0}$ of $V, W$ respectively such that $v_{1}, \ldots, v_{s}, v_{1}^{\prime}, \ldots, v_{s}^{\prime} \in V_{0}$ and $u_{1}, \ldots, u_{t}, u_{1}^{\prime}, \ldots, u_{t}^{\prime} \in W_{0}$ and such that the bilinear form $($,$) restricted$ to $V_{0} \times W_{0}$ is nondegenerate. Also $V_{0}$ and $W_{0}$ are of the same dimension, say of dimension $n$. Augment $\left\{v_{1}, \ldots, v_{s}\right\}$ to a basis $\left\{v_{1}, \ldots, v_{s}, v_{s+1}, \ldots, v_{n}\right\}$ for $V_{0}$.

By Fact 1 , there exist $x_{1}, \ldots, x_{t} \in V_{0}$ such that $\left(x_{i}, u_{j}\right)=\delta_{i j}$ for $i, j=$ $1, \ldots, t$. For each $s<k \leq n$, define $v_{k}^{\prime}=\sum_{l=1}^{t}\left(v_{k}, u_{l}^{\prime}\right) x_{l}$. Then for $s<k \leq n$ and for $j=1, \ldots, t$,

$$
\left(v_{k}^{\prime}, u_{j}\right)=\left(\sum_{l=1}^{t}\left(v_{k}, u_{l}^{\prime}\right) x_{l}, u_{j}\right)=\left(v_{k}, u_{j}^{\prime}\right) .
$$

Together with the originally given $v_{1}^{\prime}, \ldots, v_{s}^{\prime}$, we have that

$$
\left(v_{i}^{\prime}, u_{j}\right)=\left(v_{i}, u_{j}^{\prime}\right) \text { for } i=1, \ldots, n \text { and for } j=1, \ldots, t \text {. }
$$

By Fact 1 again, pick a basis $\left\{w_{1}, \ldots, w_{n}\right\}$ for $W_{0}$ such that $\left(v_{i}, w_{j}\right)=$ $\delta_{i j}$ for $i, j=1, \ldots, n$. Now we define our desired $a \in S_{W}(V)$ by $v a=$ $\sum_{i=1}^{n}\left(v, w_{i}\right) v_{i}^{\prime}$. Observe that $v_{i} a=\left(v_{i}, w_{i}\right) v_{i}^{\prime}=v_{i}^{\prime}(i=1, \ldots, n)$. So it suffices to prove that $a^{*} u_{j}=u_{j}^{\prime}$ for $j=1, \ldots, t$.

Given $u_{j}(1 \leq j \leq t)$, compute $\left(v_{i}, a^{*} u_{j}\right)=\left(v_{i} a, u_{j}\right)=\left(v_{i}^{\prime}, u_{j}\right)$ for $i=$ $1, \ldots, n$. Since $v_{i}^{\prime}(i=1, \ldots, n)$ are chosen so that $\left(v_{i}^{\prime}, u_{j}\right)=\left(v_{i}, u_{j}^{\prime}\right)$ for $j=1, \ldots, t$, we have $\left(v_{i}, a^{*} u_{j}\right)=\left(v_{i}^{\prime}, u_{j}\right)=\left(v_{i}, u_{j}^{\prime}\right)$. So $\left(v_{i}, a^{*} u_{j}-u_{j}^{\prime}\right)=0$ for $i=1, \ldots, n$. Hence $\left(V_{0}, a^{*} u_{j}-u_{j}^{\prime}\right)=0$. Note that $a^{*}$ is defined by $a^{*} u=\sum_{i=1}^{n} w_{i}\left(v_{i}^{\prime}, u\right) \in W_{0}$ for any $u \in W$. So, in particular, $a^{*} u_{j} \in W_{0}$ and hence also $a^{*} u_{j}-u_{j}^{\prime} \in W_{0}$. Since the bilinear form (, ) restricted to $V_{0} \times W_{0}$ is nondegenerate, we have $a^{*} u_{j}-u_{j}^{\prime}=0$ as desired.

Let $D$ be a division ring endowed with involution ${ }^{*}$ and let ${ }_{D} V$ be a left vector space over $D . V$ can also be regarded as a right vector space by the right scalar multiplication defined by $v \alpha=\alpha^{*} v$, where $v \in V$ and $\alpha \in D$. Note that $A\left({ }_{D} V\right)=A\left(V_{D}\right)$. For this reason, the action of $a \in A\left(V_{D}\right)$ on $V_{D}$ will also be written on the right-hand side. Suppose that there exists a nondegenerate bilinear form on ${ }_{D} V \times V_{D}$. Then ${ }_{D} V$ is said to be a self-dual space with respect to $($,$) .$ 
The *-version of the Jacobson theorem is the following well-known:

Kaplansky theorem $[1, \mathrm{p} .17]$. Let $R$ be a prime ring with involution ${ }^{*}$ and with $\operatorname{Soc}(R) \neq 0$. Then there exists a vector space $V$ over a division ring $D$ endowed with involution, which is self-dual with respect to a Hermitian or alternate bilinear form $(,$,$) in such a way that$

1. $S_{V}(V) \subseteq R \subseteq L_{V}(V)$,

2. the ${ }^{*}$ of $R$ is the adjoint of $R$ relative to this bilinear form (, ).

Furthermore, the Hermitian and alternate cases are mutually exclusive, occurring according as there is, or is not, a minimal symmetric idempotent $e^{2}=e=$ $e^{*}$.

Conversely, any $R$ such that $S_{V}(V) \subseteq R \subseteq L_{V}(V)$ and such that $R^{*}=R$ must be a prime ring with involution * and with $\operatorname{Soc}(R)=S_{V}(V) \neq 0$.

Proposition 4. Suppose that $R$ is a prime ring with involution ${ }^{*}$ and that $f$ is a *-generalized polynomial. If $f$ vanishes on a nonzero ideal of $R$, then $f$ vanishes on $Q$, the two-sided quotient ring of $R$.

Proof. Suppose that $f$ vanishes on a nonzero ideal $I$ of $R$. Replacing $I$ by $I \cap I^{*}$, we may assume from the start that $I$ is a $*$-ideal of $R$. We may also assume that $f$ is nontrivial. By Theorem 9 [8, p. 477], $I$ satisfies an ordinary nontrivial generalized polynomial identity (without ${ }^{*}$ ) and hence so does $R$. By Martindale's theorem, $R C$ is a primitive ring with nonzero socle. By Kaplansky's theorem, $S_{V}(V) \subseteq R C \subseteq L_{V}(V)$ for a self-dual vector space $V$ over a division ring $D$ endowed with involution. By Proposition 2, the two-sided Martindale quotient ring of $R C$ is $L_{V}(V)$. Note that the two-sided Martindale quotient ring $L_{V}(V)$ of $R C$ includes the two-sided Martindale quotient ring $Q$ of $R$ as a subring in a natural way. It suffices to show that $f$ vanishes on $L_{V}(V)$. Note that $\operatorname{Soc}(R C)=S_{V}(V)$. By Proposition 3, it suffices to show that $f$ vanishes on $\operatorname{Soc}(R C)$ : Suppose that $f$ vanishes on $\operatorname{Soc}(R C)$. Let $q_{1}, q_{2}, \ldots \in L_{V}(V)$ and let $v \in V$. In computing $v f\left(q_{1}, q_{2}, \ldots\right)=v^{\prime}$, we need only finitely many relations of the form $u q_{i}=u^{\prime}, q_{i}^{*} w=w^{\prime}$, where $u, u^{\prime}, w, w^{\prime} \in V$. Obviously, $\left(u^{\prime}, w\right)=\left(u q_{i}, w\right)=\left(u, q_{i}^{*} w\right)=\left(u, w^{\prime}\right)$. Proposition 3 asserts that there exists $\tilde{q}_{i} \in \operatorname{Soc}(R C)$ such that $u \tilde{q}_{i}=u^{\prime}$ and $\left(\tilde{q}_{i}\right)^{*} w=$ $w^{\prime}$. Hence $v^{\prime}=v f\left(q_{1}, q_{2}, \ldots\right)=v f\left(\tilde{q}_{1}, \tilde{q}_{2}, \ldots\right)=0$.

If $C$ is finite, then there is a nonzero *-ideal $J$ of $R$ such that $\alpha J \subseteq R$ for all $\alpha \in C$. Then $I \supseteq I J C$. But $I J C$ is a nonzero ideal of $R C$ and hence must contain the minimal ideal $\operatorname{Soc}(R C)$. So $f$ vanishes on $\operatorname{Soc}(R C)$ as desired.

Now let $C$ be infinite. If ${ }^{*}$ is of the second kind, then $R$ is special by Proposition 1. The assertion follows from the well-known corresponding result for ordinary generalized polynomial identities $\left(\right.$ without ${ }^{*}$ ). So we may assume that ${ }^{*}$ is of the first kind. We proceed by induction on the height of $f$. Pick sufficiently but finitely many $\alpha \in C$ and let $J$ be a nonzero *-ideal of $R$ such that $\alpha J \subseteq I$ for these $\alpha$ picked. Replace each indeterminate $x$ in $f$ by $\alpha x$ 
for these $\alpha$ picked. Then the resulting *-generalized polynomials vanish on $J$. Note that these $\alpha \in C$ picked are symmetric and hence can be moved out of * operation. By the Vandermonde determinant argument, we can solve these *-generalized identities for the homogeneous parts of $f$. So each homogeneous part of $f$ vanishes on $J$. It suffices to show that each homogeneous part of $f$ vanishes on $Q$. Note that the height of each homogeneous part of $f$ is not larger than that of $f$. Replacing $f$ by one of its homogeneous parts, we may assume from the start that $f$ is homogeneous in each indeterminate it involves.

Assume that the height of $f$ is 0 . Then since $f$ is homogeneous, $f$ must be multilinear. It is obvious that $f$ vanishes on $I C$. Since $I C$, a nonzero ideal of $R C$, must contain the minimal ideal $\operatorname{Soc}(R C), f$ also vanishes on $\operatorname{Soc}(R C)$ as desired. So we assume that the height of $f$ is larger than 0 . As the induction hypothesis, we also assume that the assertion of the proposition holds for any $*$-generalized polynomials with less height.

Given any indeterminate $x$ in $f$, we suppress all indeterminates other than $x$ and write $f(x)$ for $f$ for simplicity of notation. Now consider $g(x+y)=f(x+y)-f(x)-f(y)$, where $y$ is a new indeterminate. Since $g$ is of less height than $f, g$ must vanish on $Q$ by our induction hypothesis. So $f(x+y)=f(x)+f(y)$. We have thus shown that $f$ is additive on $Q$ with respect to each indeterminate it involves.

Now write $f=f\left(x_{1}, \ldots, x_{n}\right)$, where $x_{1}, \ldots, x_{n}$ are all the indeterminates $f$ involves. Set $x_{i}=\sum_{j} r_{j}^{(i)} \alpha_{j}^{(i)}$, where $r_{j}^{(i)} \in I$ and $\alpha_{j}^{(i)} \in C$. Using the additivity of $f$ on $Q$, we compute

$$
\begin{aligned}
f\left(x_{1}, x_{2}, \ldots, x_{n}\right) & =f\left(\sum_{j} r_{j}^{(1)} \alpha_{j}^{(1)}, \ldots, \sum_{j} r_{j}^{(n)} \alpha_{j}^{(n)}\right) \\
& =\sum_{j_{1} \ldots, j_{n}} f\left(r_{j_{1}}^{(1)} \alpha_{j_{1}}^{(1)}, \ldots, r_{j_{n}}^{(n)} \alpha_{j_{n}}^{(n)}\right) \\
& =\sum_{j_{1}, \ldots, j_{n}}\left(\alpha_{j_{1}}^{(1)}\right)^{h_{1}} \cdots\left(\alpha_{j_{n}}^{(n)}\right)^{h_{n}} f\left(r_{j_{1}}^{(1)}, \ldots, r_{j_{n}}^{(n)}\right),
\end{aligned}
$$

where $h_{i}=$ the $x_{i}$-degree of $f(i=1, \ldots, n)$. Since $r_{j_{l}}^{(i)} \in I, f\left(r_{j_{1}}^{(1)}, \ldots, r_{j_{n}}^{(n)}\right)$ $=0$ and hence $f\left(x_{1}, \ldots, x_{n}\right)=0$. But $x_{i}=\sum_{j} r_{j}^{(i)} \alpha_{j}^{(i)}$ are typical elements of $I C$. So $f$ vanishes on $I C$. Since $I C$, a nonzero ideal of $R C$, must contain the minimal ideal $\operatorname{Soc}(R C), f$ vanishes on $\operatorname{Soc}(R C)$ as desired.

One might be wondering whether the two-sided Martindale quotient rings respectively of $R$ and of $R C$ are equal or not. If $R$ satisfies a nontrivial polynomial identity (over $C$ ), then $R_{F}, Q$, the left Martindale quotient ring of $R C$, and the two-sided Martindale quotient ring of $R C$ are all equal to $R C$. But, as the following example shows, this is actually false in general, even if $R$ satisfies a nontrivial generalized identity. 
Example. Let $D$ be a (commutative) principal ideal domain and let $C$ be its quotient field. Suppose that ${ }_{C} V$ is an infinite-dimensional vector space over $C$. Fix a basis $\mathscr{B}=\left\{v_{1}, v_{2}, \ldots\right\}$ of ${ }_{C} V$. For $v=\sum_{i} \alpha_{i} v_{i}, u=\sum_{i} \beta_{i} v_{i} \in V$, where $v_{i} \in \mathscr{B}$ and where $\alpha_{i}, \beta_{i} \in C$ vanish for all but finitely many $i$, we define $(v, u)=\sum_{i} \alpha_{i} \beta_{i}$. Then (, ) is a nondegenerate bilinear form on $V \times V$. As usual, let $A(V)=\operatorname{End}\left({ }_{C} V\right)$. For $a \in A(V)$ such that $v_{i} a=\sum_{j} \alpha_{i j} v_{j}$, where $v_{i}, v_{j} \in \mathscr{B}, \alpha_{i j} \in C$, the adjoint of $a$, if it exists, is defined by $v_{j} a^{*}=\sum_{i} \alpha_{i j} v_{i}$. Hence, $L_{V}(V)$ consists of all $a \in A(V)$ such that for each $v_{j} \in \mathscr{B},\left(v_{i} a, v_{j}\right)=0$ for all but finitely many $i$, and $S_{V}(V)$ consists of all $a \in A(V)$ such that $v_{i} a=0$ for all but finitely many $i$. Let $D_{\infty}$ consist of all $a \in A(V)$ such that $\left(v_{i} a, v_{j}\right) \in D$ for all $v_{i}, v_{j} \in \mathscr{B}$. Define $R=S_{V}(V) \cap D_{\infty}$. Then $R C=S_{V}(V)$. Also, ideals of $R$ assume the form $d R$ for some $d \in D$. Hence the left Martindale quotient ring $R_{F}$ of $R$ and the two-sided Martindale quotient ring $Q$ of $R$ are equal to $\left\{a / d: a \in D_{\infty}\right.$ and $\left.0 \neq d \in D\right\}$ and $R_{F} \cap L_{V}(V)$, respectively. So, if $D$ is chosen so that $D \neq C$, then $A(V) \neq R_{F}$ and $L_{V}(V) \neq Q$.

\section{Proof of Main Theorem}

We begin with the following.

Lemma 8. Suppose that $R$ satisfies a nontrivial $*$-differential identity. Then $R$ satisfies a nontrivial generalized identity (without ${ }^{*}$ ).

Proof. By linearization, we may assume that $R$ satisfies a nontrivial multilinear *-differential identity $\phi\left(x_{i}^{\Delta_{j}},\left(x_{i}^{\Delta_{j}}\right)^{*}\right)=0$. Assume toward a contradiction that $R$ does not satisfy any nontrivial generalized identities (without ${ }^{*}$ ). By Theorem 9 [8, p. 77], $R$ does not satisfy any nontrivial $*$-generalized identities (without derivations) either. In particular, any linear $*$-generalized identities of $R$ are trivial. Hence Lemma 4 applies and gives that $\phi\left(z_{i j}, y_{i j}\right)=0$ is a multilinear generalized identity of $R$. Since $\phi\left(x_{i}^{\Delta_{j}},\left(x_{i}^{\Delta_{j}}\right)^{*}\right)=0$ is not trivial, $\phi\left(z_{i j}, y_{i j}\right)=0$ cannot be trivial either. This is absurd.

If every *-differential identity of $R$ is trivial, then our Main Theorem holds trivially and there is nothing to prove. So from now on, we assume that $R$ satisfies a nontrivial $*$-differential identity. By Lemma 8 above, $R$ satisfies $a$ nontrivial generalized identity.

We recall the following [4, p. 68]:

Fact 4. Assume that $R$ satisfies a nontrivial generalized identity. Let $\mu \in$ $D+D_{\text {int }}$ be such that $\mu(C)=0$. Then $\mu \in D_{\text {int }}$.

If $C$ is finite, then $\mu(C)=0$ for any $\mu \in D+D_{\text {int }}$. Hence by the identity (3), any $*$-differential identity can be brought to a $*$-generalized identity without derivations. So our Main Theorem holds trivially in this case. Thus we may assume henceforth that $C$ is infinite. By Proposition 1 and Lemma 1, we can further assume that ${ }^{*}$ is of the first kind. 
We recall the following definition [4, p. 64, fourth line from the bottom]: For $\mu_{1}, \ldots, \mu_{n} \in D+D_{\text {int }}$, we say that $\mu_{1}, \ldots, \mu_{n}$ are strongly independent if, for any $\alpha_{i} \in C, \sum \mu_{i} \alpha_{i} \in D_{\text {int }}$ implies $\alpha_{1}=\alpha_{2}=\cdots=\alpha_{n}=0$.

Lemma 9. Suppose that $\mu_{1}, \ldots, \mu_{n} \in D+D_{\text {int }}$ are strongly independent and that $a_{1}, \ldots, a_{n} \in R_{F}$. If for all $\alpha \in C, \alpha^{\mu_{1}} a_{1}+\cdots+\alpha^{\mu_{n}} a_{n}=0$, then $a_{1}=$ $\cdots=a_{n}=0$.

Proof. Suppose not. Without loss of generality, we may assume that $a_{1} \neq 0$. Choose a basis $v_{1}=a_{1}, v_{2}, \ldots$ for the subspace $\sum_{i=1}^{n} a_{i} C$. Express $a_{i} \quad(i=$ $1, \ldots, n)$ in terms of the basis $v_{1}, v_{2}, \ldots: a_{1}=v_{1}$ and $a_{i}=\beta_{i} v_{1}+\cdots$ for $i \geq 2$. Substituting these expressions into $\alpha^{\mu_{1}} a_{1}+\cdots+\alpha^{\mu_{n}} a_{n}=0$ and collecting terms according to $v_{1}, v_{2}, \ldots$, we obtain

$$
\left(\alpha^{\mu_{1}}+\alpha^{\mu_{2}} \beta_{2}+\alpha^{\mu_{3}} \beta_{3}+\cdots\right) v_{1}+(\cdot) v_{2}+\cdots=0 .
$$

Since $v_{1}, v_{2}, \ldots$ are $C$-independent, $\alpha^{\mu_{1}}+\alpha^{\mu_{2}} \beta_{2}+\alpha^{\mu_{2}} \beta_{3}+\cdots=0$ follows. Hence the derivation $\mu_{1}+\mu_{2} \beta_{2}+\mu_{3} \beta_{3}+\cdots$ vanishes on $C$ and, by Fact 4 , must be in $D_{\text {int }}$. This contradicts the strong independence of $\mu_{1}, \mu_{2}, \ldots, \mu_{n}$.

Lemma 10. If $\phi$ is a linear $*$-differential identity, then the Main Theorem holds.

Proof. $\phi$ may be written in the form

$$
\phi=\phi\left(x^{\Delta_{i}},\left(x^{\Delta_{i}}\right)^{*}\right)=\sum_{i} \sum_{k=1}^{n_{i}}\left(a_{i}^{(k)} x^{\Delta_{i}} b_{i}^{(k)}+c_{i}^{(k)}\left(x^{\Delta_{i}}\right)^{*} d_{i}^{(k)}\right),
$$

where $\Delta_{i}$ are distinct regular words in $M$. Assume that $\Delta_{1}$ is the greatest among $\Delta_{i}$. We prove by induction on $\Delta_{1}$ that if $\phi\left(x^{\Delta_{i}},\left(x^{\Delta_{i}}\right)^{*}\right)$ vanishes on a nonzero ideal of $R$, then $\phi\left(y_{i}, y_{i}^{*}\right)=0$ is a $*$-generalized identity on $R$, where $y_{i}$ are new distinct indeterminates.

If $\Delta_{1}=\varnothing$, then $\phi$ is simply a $*$-generalized polynomial (without derivations). The result follows from Proposition 4. So let us assume that $\Delta_{1} \neq \varnothing$. As the induction hypothesis, we also assume that the assertion of this lemma holds for any linear $*$-differential identity whose leading word is strictly less than $\Delta_{1}$.

Write $\Delta_{i}=\delta_{1}^{(i)} \delta_{2}^{(i)} \cdots$ and assume that $\delta_{1}^{(i)}=\delta_{2}^{(i)}=\cdots=\delta_{s_{i}}^{(i)} \neq \delta_{s_{i}+1}^{(i)}$, $0<s_{i}<p \quad(p=\operatorname{ch} R)$. We also set $\bar{\Delta}_{i}=\delta_{2}^{(i)} \delta_{3}^{(i)} \cdots$. Let $\beta \in C$. Using the formula (1) (in the proof of Lemma 3) and the fact that $\alpha^{*}=\alpha$ for any $\alpha \in C$, we have

$$
a_{i}^{(k)}(\beta x)^{\Delta_{i}} b_{i}^{(k)}=a_{i}^{(k)} \beta x^{\Delta_{i}} b_{i}^{(k)}+s_{i} a_{i}^{(k)} \beta^{\delta_{1}^{(1)}} x^{\bar{\Delta}_{i}} b_{i}^{(k)}+\cdots
$$

and

$$
c_{i}^{(k)}\left((\beta x)^{\Delta_{i}}\right)^{*} d_{i}^{(k)}=c_{i}^{(k)} \beta\left(x^{\Delta_{i}}\right)^{*} d_{i}^{(k)}+s_{i} c_{i}^{(k)} \beta^{\delta_{1}^{(\prime)}}\left(x^{\bar{\Delta}_{1}}\right)^{*} d_{i}^{(k)}+\cdots
$$


Hence

$$
\begin{aligned}
\phi(\beta x)= & \beta \sum_{i} \sum_{k=1}^{n_{i}}\left(a_{i}^{(k)} x^{\Delta_{i}} b_{i}^{(k)}+c_{i}^{(k)}\left(x^{\Delta_{i}}\right)^{*} d_{i}^{(k)}\right) \\
& +\sum_{i} \sum_{k=1}^{n_{i}} s_{i} \beta^{\delta_{1}^{(i)}}\left(a_{i}^{(k)} x^{\bar{\Delta}_{i}} b_{i}^{(k)}+c_{i}^{(k)}\left(x^{\bar{\Delta}_{i}}\right)^{*} d_{i}^{(k)}\right)+\cdots \\
= & \beta \phi(x)+\sum_{i} \sum_{k=1}^{n_{i}} s_{i} \beta^{\delta_{1}^{(i)}}\left(a_{i}^{(k)} x^{\bar{\Delta}_{i}} b_{i}^{(k)}+c_{i}^{(k)}\left(x^{\bar{\Delta}_{i}}\right)^{*} d_{i}^{(k)}\right)+\cdots
\end{aligned}
$$

Let $I$ be a nonzero ideal of $R$ on which $\phi$ vanishes. Pick a nonzero ideal $J$ of $R$ such that $J \subseteq I$ and $\beta J \subseteq I$. Then $\phi(\beta x)-\beta \phi(x)$ vanishes on $J$. Note that the leading word $\bar{\Delta}_{1}$ of $\phi(\beta x)-\beta \phi(x)$ is strictly smaller than $\Delta_{1}$. So the induction hypothesis applies.

We want to collect the terms in $\phi(\beta x)-\beta \phi(x)$ which contain the regular derivation word $\bar{\Delta}_{1}$ : Let $\mu_{1}, \ldots, \mu_{n}$ be all derivations in $M$ other than $\delta_{1}^{(1)}$ such that $\mu_{1} \bar{\Delta}_{1}, \ldots, \mu_{n} \bar{\Delta}_{1}$ occur in $\phi$. Let us say $\mu_{1} \bar{\Delta}_{1}=\Delta_{r_{1}}, \ldots, \mu_{n} \bar{\Delta}_{1}=\Delta_{r_{n}}$. Then the sum of those terms in $\phi(\beta x)-\beta \phi(x)$ containing $\bar{\Delta}_{1}$ is of the form

$$
\begin{aligned}
s_{1} \beta^{\delta_{1}^{(1)}} & \sum_{k=1}^{n_{1}}\left(a_{1}^{(k)} x^{\bar{\Delta}_{1}} b_{1}^{(k)}+c_{1}^{(k)}\left(x^{\bar{\Delta}_{1}}\right)^{*} d_{1}^{(k)}\right) \\
& +\sum_{\tau=1}^{n} \beta^{\mu_{\tau}}\left(\sum_{k=1}^{n_{r_{\tau}}}\left(a_{r_{\tau}}^{(k)} x^{\bar{\Delta}_{1}} b_{r_{\tau}}^{(k)}+c_{r_{\tau}}^{(k)}\left(x^{\bar{\Delta}_{1}}\right)^{*} d_{r_{\tau}}^{(k)}\right)\right) .
\end{aligned}
$$

By the induction hypothesis,

$$
\begin{aligned}
& s_{1} \beta^{\delta_{1}^{(1)}} \sum_{k=1}^{n_{1}}\left(a_{1}^{(k)} y b_{1}^{(k)}+c_{1}^{(k)} y^{*} d_{1}^{(k)}\right) \\
& +\sum_{\tau=1}^{n} \beta^{\mu_{\tau}}\left(\sum_{k=1}^{n_{r_{\tau}}}\left(a_{r_{\tau}}^{(k)} y b_{r_{\tau}}^{(k)}+c_{r_{\tau}}^{(k)} y^{*} d_{r_{\tau}}^{(k)}\right)\right)=0
\end{aligned}
$$

is a *-generalized identity for $R$. Since this holds for any $\beta \in C$ and since $\delta_{1}^{(1)}, \mu_{1}, \ldots, \mu_{n}$ are strongly independent, by Lemma 9 ,

$$
\sum_{k=1}^{n_{1}}\left(a_{1}^{(k)} y b_{1}^{(k)}+c_{1}^{(k)} y^{*} d_{1}^{(k)}\right)=0
$$

is a $*$-generalized identity for $R$. Hence

$$
\phi_{1}=\sum_{i \geq 2} \sum_{k=1}^{n_{i}}\left(a_{i}^{(k)} x^{\Delta_{i}} b_{i}^{(k)}+c_{i}^{(k)}\left(x^{\Delta_{i}}\right)^{*} d_{i}^{(k)}\right)
$$

also vanishes on $I$. The leading word of $\phi_{1}$ is obviously smaller than that of $\phi$. So by the induction hypothesis again,

$$
\sum_{i \geq 2} \sum_{k=1}^{n_{i}}\left(a_{i}^{(k)} y_{i} b_{i}^{(k)}+c_{i}^{(k)} y_{i}^{*} d_{i}^{(k)}\right)=0
$$


is also a $*$-generalized identity for $R$. Combining this with the identity

$$
\sum_{k=1}^{n_{1}}\left(a_{1}^{(k)} y b_{1}^{(k)}+c_{1}^{(k)} y^{*} d_{1}^{(k)}\right)=0,
$$

the desired result follows.

Using a proof similar to that of Lemma 4, we have

Lemma 11. If $\phi$ is a multilinear $*$-differential identity, then the Main Theorem holds.

Using Lemma 11 and arguing as in the proof of Lemma 6, we have the following

Lemma 12. If $\operatorname{ch} R=0$, then the Main Theorem holds.

By Lemma 11, we may further assume henceforth that $\operatorname{ch} R=p>0$, where $p$ is a fixed prime.

Since $R$ satisfies a nontrivial generalized identity, by Martindale's theorem [7], the socle of $R C$ is nonzero. Set $\sigma=\operatorname{Soc}(R C)$. By the Kaplansky theorem, there exists a self-dual space ${ }_{D} V$ over a division ring $D$ endowed with involution such that

$$
S_{V}(V) \subseteq R C \subseteq L_{V}(V)
$$

and such that $\sigma=S_{V}(V)$. Note that $V_{R C}$ is an irreducible right $R C$-module and $D=\operatorname{Hom}\left(V_{R C}, V_{R C}\right)$. Since $\operatorname{Soc}(R C) \neq 0$, all the irreducible right $R C$ modules are isomorphic. The division ring $D$ is also unique up to isomorphism and is thus called the skew field of $R C$. Note that, by [7], $D$ is finite dimensional over $C$.

We need the following from $[4$, p. 74]:

Fact 5. Let $I$ be a nonzero ideal of $R$. Then $I C^{p} \supseteq \sigma$.

Note that the coefficients of $*$-differential polynomials of $R$ are allowed to lie in $R_{F}$. Since the left Martindale quotient ring of $R C$ includes $R_{F}$ as a subring in a natural way and since, by Proposition 2, both the left Martindale quotient ring of $R C$ and the left Martindale quotient ring of $\operatorname{Soc}(R C)$ are equal to $A(V)$, any *-differential polynomial of $R$ can be naturally regarded as a $*$-differential polynomial of $\operatorname{Soc}(R C)$. The following lemma is stated in this sense.

Lemma 13. If $\phi$ is a $*$-differential identity of $R$, then $\phi$ is also a $*$-differential identity of $\sigma$.

Proof. We prove by induction on the height of $\phi$ that if $\phi$ vanishes on a nonzero *-ideal $I$ of $R$, then $\phi$ vanishes on $J C^{p}$ for some nonzero ideal $J$ of $R$. Our desired result will follow immediately from Fact 5 .

As in the proof of Proposition 4, pick sufficiently but finitely many $\alpha \in C$. Let $J$ be a nonzero *-ideal of $R$ such that $\alpha J \subseteq I$ for those $\alpha$ picked. Replace each indeterminate $x$ in $\phi$ by $\alpha^{p} x$ for those $\alpha \in C$ picked. Note that the 
resulting $*$-differential polynomials after such replacement vanish on $J$. Note that $\alpha^{p}$ are constants of derivations and hence can be moved out of derivations. Using the Vandermonde determinant argument, we can solve these $*$-differential identities of $J$ for the homogeneous parts of $\phi$. So each homogeneous part of $\phi$ vanishes on $J$. Note that the height of each homogeneous part of $\phi$ is less than or equal to that of $\phi$. Replacing $\phi$ by one of its homogeneous parts (and $I$ by $J$ ), we may assume from the start that $\phi$ is homogeneous in each indeterminate it involves.

If the height of $\phi$ is 0 , then $\phi$ must be multilinear. If $\phi$ vanishes on $I$, then obviously $\phi$ also vanishes on $I C^{p}$ as desired. So we assume that the height of $\phi$ is larger than 0 . As our induction hypothesis, we also assume that the assertion holds for any $*$-differential polynomial of less height.

Let $x$ be an indeterminate occurring in $\phi$. Suppressing indeterminates other than $x$, write $\phi=\phi(x)$ for simplicity of notation. Let $y$ be a new indeterminate not occurring in $\phi$. The $*$-differential polynomial $\phi(x+y)-\phi(x)-\phi(y)$ also vanishes on $I$. Since the height of $\phi(x+y)-\phi(x)-\phi(y)$ is strictly less than that of $\phi$, by our induction hypothesis, there is a nonzero ideal $J$ of $R$ such that $\phi(x+y)-\phi(x)-\phi(y)$ vanishes on $J C^{p}$. We have thus shown that $\phi$ is additive on $J C^{p}$ with respect to $x$.

Let $x_{1}, \ldots, x_{n}$ be all indeterminates occurring in $\phi$. Write

$$
\phi=\phi\left(x_{1}, \ldots, x_{n}\right) .
$$

By the paragraph above, for each $i(i=1, \ldots, n)$, there exists a nonzero ideal $J_{i}$ such that $\phi$ is additive on $J_{i} C^{p}$ with respect to $x_{i}$. Set $K=I \cap\left(\bigcap_{i=1}^{n} J_{i}\right)$. Assume that the $x_{i}$-degree of $\phi$ is $h_{i}$. Set $x_{i}=\sum_{j} r_{j}^{(i)} \alpha_{j}^{(i)}$ where $r_{j}^{(i)} \in K$ and $\alpha_{j}^{(i)} \in C^{p}$. Using the additivity and the homogeneity of $\phi$, we compute

$$
\begin{aligned}
& \phi\left(\sum_{j} r_{j}^{(1)} \alpha_{j}^{(1)}, \sum_{j} r_{j}^{(2)} \alpha_{j}^{(2)}, \ldots, \sum_{j} r_{j}^{(n)} \alpha_{j}^{(n)}\right) \\
& \quad=\sum_{j_{1}, \ldots, j_{n}} \phi\left(r_{j_{1}}^{(1)} \alpha_{j_{1}}^{(1)}, r_{j_{2}}^{(2)} \alpha_{j_{2}}^{(2)}, \ldots, r_{j_{n}}^{(n)} \alpha_{j_{n}}^{(n)}\right) \\
& \quad=\sum_{j_{1}, \ldots, j_{n}}\left(\alpha_{j_{1}}^{(1)}\right)^{h_{1}}\left(\alpha_{j_{2}}^{(2)}\right)^{h_{2}} \ldots\left(\alpha_{j_{n}}^{(n)}\right)^{h_{n}} \phi\left(r_{j_{1}}^{(1)}, r_{j_{2}}^{(2)}, \ldots, r_{j_{n}}^{(n)}\right) \\
& \quad=0 .
\end{aligned}
$$

Hence $\phi$ vanishes on $K C^{p}$ as desired.

By the Jacobson-Noether theorem, the skew field $D$ of $R$ possesses a maximal subfield $\Phi$ which is separable over $C$. $\Phi$ is hence a primitive extension of $C$. Assume that $\Phi=C(v)$, where $v$ is a primitive element of $\Phi$ over $C$ satisfying the minimal polynomial

$$
f(x)=\sum_{k} \alpha_{k} x^{k}
$$


By the separability of $\Phi$ over $C, f^{\prime}(v)=\sum_{k} k \alpha_{k} v^{k-1} \neq 0$.

Any derivation $\mu$ on $C$ can be uniquely extended to $\Phi(=C(v))$ by defining

$$
v^{\mu}=-\left(\sum \alpha_{k}^{\mu} v^{k}\right)\left(f^{\prime}(v)\right)^{-1}
$$

Now consider the ring $\sigma \otimes_{C} \Phi$. Any derivation $\mu$ on $\sigma$ can be extended to $\sigma \otimes_{C} \Phi$ by defining

$$
(r \otimes \alpha)^{\mu}=r^{\mu} \otimes \alpha+r \otimes \alpha^{\mu}
$$

where $r \in \sigma$ and $\alpha \in \Phi$. The involution ${ }^{*}$ on $\sigma$ can also be extended to $\sigma \otimes_{C} \Phi$ by defining

$$
(r \otimes \alpha)^{*}=r^{*} \otimes \alpha
$$

where $r \in \sigma$ and $\alpha \in \Phi$.

Since the left Martindale quotient rings of $\sigma$ is a subring of the left Martindale quotient ring of $\sigma \otimes_{C} \Phi$ in a natural way, by means of the above extensions of derivations and involution on $\sigma$ to $\sigma \otimes_{C} \Phi$, any *-differential polynomial of $\sigma$ can be naturally interpreted as a $*$-differential polynomial of $\sigma \otimes_{C} \Phi$. The following lemma is stated in this sense.

Lemma 14. If $\phi$ is a *-differential identity on $\sigma$, then $\phi$ is also a *-differential identity on $\sigma \otimes_{C} \Phi$.

Proof. Let $\phi=\phi\left(x_{i}^{\Delta_{j}},\left(x_{i}^{\Delta_{j}}\right)^{*}\right)=0$ be a $*$-differential identity for $\sigma$. Using identity $(9)^{\prime}$ to bring ${ }^{*}$ inside of derivations, we have $\phi=\phi\left(x_{i}^{\Delta_{j}},\left(x_{i}^{*}\right)^{\Delta_{j}^{*}}\right)=0$. Suppressing derivation words for simplicity of notation, we write $\phi=\phi\left(x_{i}, x_{i}^{*}\right)$. Set $x_{i}=\sum_{j} a_{i j} \eta_{i j}$, where $a_{i j}$ are fixed elements of $\sigma$ and $\eta_{i j}$ are indeterminates intended to range over $C$. Since ${ }^{*}$ is of the first kind, $x_{i}^{*}=\sum_{j} a_{i j}^{*} \eta_{i j}$. Substitute these $x_{i}$ and $x_{i}^{*}$ into $\phi$ and write the resulting expression in the following form:

$$
\phi\left(\sum_{j} a_{i j} \eta_{i j}, \sum_{j} a_{i j}^{*} \eta_{i j}\right)=\sum_{b} b \phi_{b}\left(\eta_{i j}^{\Delta_{k}}\right),
$$

where $b$ are $C$-linearly independent elements in the left Martindale quotient ring of $\sigma$ and where $\phi_{b}\left(\eta_{i j}^{\Delta_{k}}\right)$ are differential polynomials in $\eta_{i j}$ with coefficients also in $C$. If $\eta_{i j}$ are assigned values from $C$, then, since $\sum a_{i j} \eta_{i j} \in \sigma$ and $\left(\sum a_{i j} \eta_{i j}\right)^{*}=\sum a_{i j}^{*} \eta_{i j} \in \sigma, \phi\left(\sum_{j} a_{i j} \eta_{i j}, \sum_{j} a_{i j}^{*} \eta_{i j}\right)$ vanishes. Since $b$ are $C$-linearly independent, $\phi_{b}\left(\eta_{i j}^{\Delta_{k}}\right)$ vanishes on $C$ for each $b$. Thus $\phi_{b}\left(\eta_{i j}^{\Delta_{k}}\right)=0$ is a differential identity (without ${ }^{*}$ ) for $C$. By the Kharchenko theorem, $\phi_{b}\left(\eta_{i j k}\right)=0$ is an identity on $C$, where $\eta_{i j k}$ are new distinct indeterminates. Since $C$ is an infinite field, $\phi_{b}\left(\eta_{i j k}\right)$ is a trivial polynomial. Thus $\phi_{b}\left(\eta_{i j}^{\Delta_{k}}\right)$ also vanishes trivially when $\eta_{i j}$ are assigned values in $\Phi$. So $\phi\left(\sum_{j} a_{i j} \eta_{i j}, \sum_{j} a_{i j}^{*} \eta_{i j}\right)$ vanishes when $\eta_{i j}$ range over $\Phi$. Since $a_{i j} \in \sigma, \eta_{i j} \in \Phi$ are arbitrary and since $\sum a_{i j} \eta_{i j}$, where $a_{i j} \in \sigma, \eta_{i j} \in \Phi$ are typical elements of $\sigma \otimes_{C} \Phi, \phi$ vanishes on $\sigma \otimes_{C} . \Phi$ as desired. 
As usual, each element in $\sigma \otimes_{C} \Phi$ can be interpreted as a linear map in $\operatorname{End}\left({ }_{\Phi} V\right)$ by defining

$$
v(r \otimes \alpha)=\alpha v r
$$

where $v \in V, r \in \sigma$, and $\alpha \in \Phi$. Such a representation is faithful by Theorem 2.2 [7, p. 504]. Obviously, $V$ is an irreducible $\sigma \otimes_{C} \Phi$ module. It is easy and well known that the commuting division ring of $\sigma \otimes_{C} \Phi$ in $\operatorname{End}\left({ }_{\Phi} V\right)$ is $\Phi$ itself. $D$ is finite dimensional over $C$ and hence must also be finite dimensional over $\Phi$. For $r \in \sigma$, the range of $r$ is finite dimensional over $D$ and hence is also finite dimensional over $\Phi$. So each element in $\sigma \otimes_{C} \Phi$ is of finite rank in $\operatorname{End}\left({ }_{\Phi} V\right)$. Thus $\sigma \otimes_{C} \Phi$ coincides with its own socle.

The bilinear form $($,$) defined on { }_{D} V$ is no longer a bilinear form on ${ }_{\Phi} V$, since the values of $($,$) fall in D$ but not in $\Phi$. However, by the Kaplansky theorem, there exists a self-dual space $U$ over a division ring $\Delta$ endowed with involution such that

$$
S_{U}(U) \subseteq \sigma \otimes_{C} \Phi \subseteq L_{U}(U)
$$

Since the socle of $\sigma \otimes_{C} \Phi$ is nonzero, all the irreducible $\sigma \otimes_{C} \Phi$ modules are isomorphic and hence so are their commuting division rings. Thus we may assume $\Delta=\Phi$. Since the two-sided Martindale quotient ring of $\sigma \otimes_{C} \Phi$ includes $R$ as a subring naturally, by Proposition 4, it suffices to prove our Main Theorem for $\sigma \otimes_{C} \Phi$. Replacing $R$ by $\sigma \otimes_{C} \Phi$, we may assume henceforth that the skew field of $\sigma$ coincides with its own extended centroid $C$.

Now are ready to give

Proof of the Main Theorem. It is well known that any $\delta \in D+D_{\text {int }}$ can be uniquely extended to a derivation on the ring $R_{F}$ of left quotients. See for example, Exercise 10 [6, p. 101]. The derivation thus extended will also be denoted by $\delta$. So we assume that any $\delta \in D+D_{\text {int }}$ is defined on the whole $R_{F}$.

Suppose that $e$ is an idempotent in $\sigma$. Then $V e$ is a finite-dimensional subspace over $C$. Let us say that $V e$ is of dimension $m$ over $C$. Choose a basis $\left\{v_{1}, \ldots, v_{m}\right\}$ for $V e$ over $C$. Also choose a basis $\left\{v_{\alpha}: \alpha>m\right\}$ for $V(1-e)$. Then $\left\{v_{\alpha}: \alpha \geq 1\right\}$ is a basis of the whole space $V$. Let $e_{\alpha \beta} \in R_{F}$ $(=A(V))$ be the linear transformation such that $v_{\alpha} e_{\alpha \beta}=v_{\beta}$ and $v_{\gamma} e_{\alpha \beta}=0$ for $\gamma \neq \alpha$. If $\alpha, \beta \leq m$, then $e_{\alpha \beta} \in e \sigma e$. Also $\left\{e_{\alpha \beta}: \alpha, \beta \leq m\right\}$ forms a basis of $e \sigma e$ over the field $C$.

For each $\delta \in D+D_{\text {int }}$, we define a new derivation $\bar{\delta}$ on $R_{F} \quad(=A(V))$ as follows: Let $a \in R_{F}$. be such that $v_{\alpha} a=\sum_{\beta} c_{\alpha \beta} v_{\beta}$. Define $a^{\bar{\delta}} \in A(V)$ by setting

$$
v_{\alpha}\left(a^{\bar{\delta}}\right)=\sum_{\beta} c_{\alpha \beta}^{\delta} v_{\beta} .
$$

Observe that $e_{\alpha \beta}^{\bar{\delta}}=0$. Also $\delta-\bar{\delta}$ acts trivially on $C$ and hence must be an inner derivation in $R_{F}$. Let $r_{\delta} \in R_{F}$ be such that $\delta=\bar{\delta}+a d\left(r_{\delta}\right)$, where 
$\operatorname{ad}\left(r_{\delta}\right)$ is the inner derivation defined by $r_{\delta} \in R_{F}$. Let $\bar{M}=\{\bar{\delta}: \delta \in M\}$. For $\delta_{1}, \delta_{2} \in M$, we define $\bar{\delta}_{1}<\bar{\delta}_{2}$ if and only if $\delta_{1}<\delta_{2}$. For $\Delta=\delta_{1} \delta_{2} \cdots \delta_{n}$, where $\delta_{i} \in M$, define $\bar{\Delta}=\bar{\delta}_{1} \bar{\delta}_{2} \ldots \bar{\delta}_{n}$. Note that if $\Delta$ is a regular word in $M$, then $\bar{\Delta}$ is also a regular word in $\bar{M}$.

Suppose that $\phi$ is a given $*$-differential identity of $R$. As in the proof of the generalized Kharchenko theorem (see also the remark following Lemma 4 ), we may assume that $\phi$ involves only one indeterminate $x$. Write $\phi=$ $\phi\left(x^{\Delta_{j}},\left(x^{\Delta_{\jmath}}\right)^{*}\right)$, where $\Delta_{j}$ are regular words in $M$. We may assume that $\Delta_{1}>$ $\Delta_{2}>\cdots$. For simplicity, let us also assume that $\left\{\Delta_{j}: j=1,2, \ldots\right\}$ is closed under subwords.

Our strategy is to replace each $\delta \in M$ in $\phi$ by the more concrete derivation $\bar{\delta}+a d\left(r_{\delta}\right)$. But the difficulty is that ${ }^{*}$ is defined only on $Q$ and not on the whole $R_{F}$. The resulting expression after such substitution may involve $\left(x^{\bar{\delta}}\right)^{*}$ and $r_{\delta}^{*}$. Even if $x$ is assumed to range over $Q, x^{\bar{\delta}}$ may fall out of $Q$. Similarly, $r_{\delta}$ may possibly not be in $Q$. In either case, the expressions $\left(x^{\bar{\delta}}\right)^{*}$ and $r_{\delta}^{*}$ make no sense.

Our solution is as follows: Using the identity (9), we bring $\phi\left(x^{\Delta_{j}},\left(x^{\Delta_{j}}\right)^{*}\right)$ to $\phi\left(x^{\Delta_{j}},\left(x^{*}\right)^{\Delta_{j}^{*}}\right)$, where $\Delta_{j}^{*}$ are regular words in $M^{*}$. Observe the following: For $\alpha \in C$ and for $\delta \in D+D_{\text {int }}$,

$$
\alpha^{\left(\delta^{*}\right)}=\left(\left(\alpha^{*}\right)^{\delta}\right)^{*}=\left(a^{\delta}\right)^{*}=\alpha^{\delta},
$$

since $^{*}$ is assumed to be of the first kind. Hence $\delta^{*}-\delta$ vanishes on $C$ and so does $\delta^{*}-\bar{\delta}$. Thus there exists $t_{\delta} \in R_{F}$ such that $\delta^{*}=\bar{\delta}+a d\left(t_{\delta}\right)$.

Now, in $\phi\left(x^{\Delta_{j}},\left(x^{*}\right)^{\Delta_{j}^{*}}\right)$, we substitute $\bar{\delta}+a d\left(r_{\delta}\right)$ for $\delta$ in $\Delta_{j}$ and $\bar{\delta}+a d\left(t_{\delta}\right)$ for $\delta^{*}$ in $\Delta_{j}^{*}$, respectively. Using identities (1)-(6), the resulting expression of such substitution can be brought to a new $*$-differential polynomial $\psi\left(x^{\bar{\Delta}_{j}},\left(x^{*}\right)^{\bar{\Delta}_{j}}\right)$, where $\bar{\Delta}_{j}$ are regular words in $\bar{M}$.

Define $h_{j}(j \geq 1)$ inductively as follows: For $j=1$, define $h_{1}=$ the highest total degree of $x^{\Delta_{1}}$ and $\left(x^{\Delta_{1}}\right)^{*}$ in $\phi$. For $j>1$, define $h_{j}=$ the highest total degree of $x^{\Delta_{j}}$ and $\left(x^{\Delta_{j}}\right)^{*}$ in those monomials of $\phi$ whose total degree of $x^{\Delta_{t}}$ and $\left(x^{\Delta_{l}}\right)^{*}$ is $h_{i}$ for each $i<j$.

We define the leading part $\phi_{0}\left(x^{\Delta_{j}},\left(x^{\Delta_{j}}\right)^{*}\right)$ of $\phi\left(x^{\Delta_{j}},\left(x^{\Delta_{j}}\right)^{*}\right)$ to be the sum of those monomials of $\phi$ whose total degree of $x^{\Delta_{j}}$ and $\left(x^{\Delta_{j}}\right)^{*}$ is $h_{j}$ for each $j \geq 1$. Similarly, we define the leading part $\psi_{0}\left(x^{\bar{\Delta}_{j}},\left(x^{*}\right)^{\bar{\Delta}_{j}}\right)$ of $\psi\left(x^{\bar{\Delta}_{j}},\left(x^{*}\right)^{\bar{\Delta}_{j}}\right)$ to be the sum of those monomials of $\psi$ whose total degree of $x^{\bar{\Delta}_{j}}$ and $\left(x^{*}\right)^{\bar{\Delta}_{j}}$ is $h_{j}$ for each $j \geq 1$. It is important that the generalized polynomial $\phi_{0}\left(z_{j}, y_{j}\right)$ coincides with the generalized polynomial $\psi_{0}\left(z_{j}, y_{j}\right)$, where $z_{j}, y_{j}$ are new indeterminates.

Set $x=\sum_{1 \leq \alpha, \beta \leq m} e_{\alpha \beta} \zeta_{\alpha \beta \beta}$, where $\zeta_{\alpha \beta \beta}$ are indeterminates intended to range over $C$ and hence are assumed to commute with $R_{F}$. Since ${ }^{*}$ is of the first 
kind, we have $x^{*}=\sum_{1 \leq \alpha, \beta \leq m} e_{\alpha \beta}^{*} \zeta_{\alpha \beta}$. Suppose that $\bar{\Delta}=\bar{\delta}_{1} \bar{\delta}_{2} \cdots \bar{\delta}_{s} \bar{\delta}_{s+1} \ldots$ is a regular word in $\bar{M}$, where $\bar{\delta}_{1}=\bar{\delta}_{2}=\cdots=\bar{\delta}_{s} \neq \bar{\delta}_{s+1}$ and $0<s<p$ $(p=\operatorname{ch} R)$. Since $e_{\alpha \beta}^{\bar{\delta}}=0$ for $\bar{\delta} \in \bar{M}$, we have

$$
x^{\bar{\Delta}}=\sum_{1 \leq \alpha, \beta \leq m} e_{\alpha \beta} \zeta_{\alpha \beta}^{\bar{\Delta}} .
$$

Using the formula (1) in the proof of Lemma 3,

$$
\begin{aligned}
\left(x^{*}\right)^{\bar{\Delta}} & =\left(\sum_{1 \leq \alpha, \beta \leq m} e_{\alpha \beta}^{*} \zeta_{\alpha \beta}\right)^{\bar{\Delta}} \\
& =\sum_{1 \leq \alpha, \beta \leq m}\left(e_{\alpha \beta}^{*} \zeta_{\alpha \beta}^{\bar{\Delta}}+s\left(e_{\alpha \beta}^{*}\right)^{\bar{\delta}_{1}} \zeta_{\alpha \beta}^{\bar{\delta}_{2} \bar{\delta}_{3} \cdots}+\cdots\right),
\end{aligned}
$$

where the dots above denote a sum of terms $\left(e_{\alpha \beta}^{*}\right)^{\bar{\Delta}^{\prime}}\left(\zeta_{\alpha \beta}\right)^{\bar{\Delta}^{\prime \prime}}$, where $\bar{\Delta}^{\prime}, \bar{\Delta}^{\prime \prime}<$ $\bar{\delta}_{2} \bar{\delta}_{3} \ldots$. Substitute these expressions for $x^{\bar{\Delta}}$ and $\left(x^{*}\right)^{\bar{\Delta}}$ into $\psi\left(x^{\bar{\Delta}_{j}},\left(x^{*}\right)^{\bar{\Delta}_{j}}\right)$ and let $\eta\left(\zeta_{\alpha \beta}^{\bar{\Delta}_{j}}\right)$ be the $*$-differential polynomial thus obtained.

We define the leading part $\eta_{0}\left(\zeta_{\alpha \beta}^{\bar{\Delta}_{j}}\right)$ to be the sum of those monomials of $\eta$ whose total degree of $\zeta_{\alpha \beta}^{\bar{\Delta}_{j}}(1 \leq \alpha, \beta \leq m)$ is $h_{j}$ for each $j \geq 1$. Let $\zeta_{\alpha \beta j}$ be new indeterminates. Observe that

$$
\begin{aligned}
\eta_{0}\left(\zeta_{\alpha \beta j}\right) & =\psi_{0}\left(\sum_{1 \leq \alpha, \beta \leq m} e_{\alpha \beta} \zeta_{\alpha \beta j}, \sum_{1 \leq \alpha, \beta \leq m} e_{\alpha \beta}^{*} \zeta_{\alpha \beta j}\right) \\
& =\phi_{0}\left(\sum_{1 \leq \alpha, \beta \leq m} e_{\alpha \beta} \zeta_{\alpha \beta j}, \sum_{1 \leq \alpha, \beta \leq m} e_{\alpha \beta}^{*} \zeta_{\alpha \beta j}\right) .
\end{aligned}
$$

Now write $\eta\left(\zeta_{\alpha \beta}^{\bar{\Delta}_{j}}\right)=\sum_{v} v \eta_{v}\left(\zeta_{\alpha \beta}^{\bar{\Delta}_{j}}\right)$, where $v \in R_{F}$ are $C$-linearly independent and where $\eta_{v}\left(\zeta_{\alpha \beta}^{\bar{\zeta}_{j}}\right)$ are differential polynomials in $\zeta_{\alpha \beta}$ with coefficients in $C$. When $\zeta_{\alpha \beta}$ are assigned values from $C, \eta\left(\zeta_{\alpha \beta}^{\bar{\Delta}_{j}}\right)$ assumes the constant value 0 . Since $v$ are assumed to be linearly $C$-independent, $\eta_{v}\left(\zeta_{\alpha \beta}^{\bar{\Delta}_{j}}\right)=0$ for each $v$. Thus, for each $v, \eta_{v}\left(\zeta_{\alpha \beta}^{\bar{\Delta}_{1}}\right)=0$ is a differential identity on $C$. By the Kharchenko theorem, $\eta_{v}\left(\zeta_{\alpha \beta j}\right)=0$ is a generalized polynomial identity on $C$, where $\zeta_{\alpha \beta \beta j}$ are new indeterminates. Hence $\eta\left(\zeta_{\alpha \beta j}\right)=0$ is also a generalized identity on $C$ (with coefficients in $R_{F}$ ). Since $C$ is assumed to be infinite, the leading part $\eta_{0}\left(\zeta_{\alpha \beta j}\right)=0$ of $\eta\left(\zeta_{\alpha \beta j}\right)=0$ is also a generalized identity on $C$. Since

$$
\eta_{0}\left(\zeta_{\alpha \beta j}\right)=\phi_{0}\left(\sum_{1 \leq \kappa, \beta \leq m} e_{\alpha \beta \beta} \zeta_{\alpha \beta j}, \sum_{1 \leq \kappa, \beta \leq m} e_{\alpha \beta \beta}^{*} \zeta_{\alpha \beta j}\right)
$$


and since $e_{\alpha \beta}(1 \leq \alpha, \beta \leq m)$ span $e \sigma e$ over $C$, we obtain that $\phi_{0}\left(y_{j}, y_{j}^{*}\right)=0$ for $y_{j} \in e \sigma e$.

Given any finitely many $y_{1}, y_{2}, \ldots \in \sigma$, there exists an idempotent $e \in \sigma$ such that $y_{1}, y_{2}, \ldots \in e \sigma e$. By the result of the previous paragraph, $\phi_{0}\left(y_{j}, y_{j}^{*}\right)$ $=0$. Thus we have shown that $\phi_{0}\left(y_{j}, y_{j}^{*}\right)=0$ is a $*$-generalized identity on $\sigma$. By Proposition $4, \phi_{0}\left(y_{j}, y_{j}^{*}\right)=0$ is also a $*$-generalized identity on $Q$.

Now consider

$$
\phi^{\prime}\left(x^{\Delta_{j}},\left(x^{\Delta_{j}}\right)^{*}\right)=\phi\left(x^{\Delta_{j}},\left(x^{\Delta_{j}}\right)^{*}\right)-\phi_{0}\left(x^{\Delta_{j}},\left(x^{\Delta_{j}}\right)^{*}\right) .
$$

Let $\phi_{0}^{\prime}\left(x^{\Delta_{j}},\left(x^{\Delta_{j}}\right)^{*}\right)$ be the leading part of $\phi^{\prime}\left(x^{\Delta_{j}},\left(x^{\Delta_{j}}\right)^{*}\right)$. Repeating the above argument, we can show similarly that $\phi_{0}^{\prime}\left(y_{j}, y_{j}^{*}\right)$ vanishes on $Q$. Continuing in this manner, we can prove finally that the Main Theorem holds for $\phi\left(x^{\Delta_{j}},\left(x^{\Delta_{j}}\right)^{*}\right)$.

Remark 1. In our Main Theorem above, derivations are assumed to be in $D_{\text {int }}+C$ Der $R$. However, as remarked in [4, p. 74], Der $R$ may be replaced by the larger set consisting of all derivations $\mu \in \operatorname{Der} R_{F}$ for which there exists a nonzero ideal $I$ of $R$ such that $I^{\mu} \subseteq R$. Also the given differential identity can be assumed to vanish only on a nonzero ideal of $R$ instead of on the whole ring $R$. The assertion of the Main Theorem above (together with its proof) still holds for such a generalization.

Remark 2. As in [3] and [4], we have deliberately avoided the following two fundamental questions: (1) With respect to a basis $M$ of $D$ linearly ordered by $<$, does a differential polynomial give rise, via the basic identities (1)-(9), to a unique differential polynomial of the form $\phi\left(x_{i}^{\Delta_{j}},\left(x_{i}^{\Delta_{j}}\right)^{*}\right)$, where $\Delta_{j}$ are distinct regular derivation words in $M$ (with respect to $<$ ) and where $\phi\left(z_{i j}, z_{i j}^{*}\right)$ is a $*$-generalized polynomial? (2) Is the nontriviality of a $*$-differential polynomial independent of the choice of $M$ and the linear order < on it? Both questions have affirmative answers. Actually, we can give a free-product treatment of *differential polynomials as initiated in [5], where all the basic notions can be made precise and then the two questions above can be proved mathematically. As such a treatment is rather long and does not seem to be immediately relevant to our main theme here, we take up these matters somewhere else.

\section{REFERENCES}

1. I. N. Herstein, Rings with involutions, Univ. of Chicago Press, Chicago, 1976.

2. N. Jacobson, Structure of rings, Amer. Math. Soc., Providence, R.I., 1964.

3. V. K. Kharchenko, Differential identities of prime rings, Algebra i Logika 17 (1978), 220-238.

4. __ Differntial identities of semiprime rings, Algebra i Logika 18 (1979), 86-119.

5. C. Lanski, Differential identities in prime rings with involution, Trans. Amer. Math. Soc. 291 (1985), 765-787.

6. J. Lambek, Lectures in rings and modules, Chelsea, 1976. 
7. W. S. Martindale III, Prime rings with involution and generalized polynomial identities, J. Algebra 22 (1972), 502-516.

8. L. H. Rowen, Generalized polynomial identities, J. Algebra 34 (1975), 458-480.

9. ___ Polynomial identities in ring theory, Academic Press, New York, 1980.

Department of Mathematics, National Taiwan University, Taipei, Taiwan 10764, RePUBlic OF China 